\title{
Comparative proteomic analysis of Ulva prolifera response to high temperature stress
}

Meihua Fan ${ }^{1 *}$, Xue Sun², Zhi Liao ${ }^{1}$, Jianxin Wang ${ }^{1}$, Yahe $\mathrm{Li}^{2}$ and Nianjun $\mathrm{Xu}^{2^{*}}$

\begin{abstract}
Background: Ulva prolifera belongs to green macroalgae and is the dominant species of green tide. It is distributed worldwide and is therefore subject to high-temperature stress during the growth process. However, the adaptation mechanisms of the response of $U$. prolifera to high temperatures have not been clearly investigated yet.

Methods: In this study, isobaric tags for relative and absolute quantitation (iTRAQ) labelling was applied in combination with the liquid chromatography-tandem mass spectrometry (LC-MS/MS) to conduct comparative proteomic analysis of the response of $U$. prolifera to high-temperature stress and to elucidate the involvement of this response in adaptation mechanisms. Differentially expressed proteins (DEPs) of U. prolifera under high temperature (denote UpHT) compared with the control (UpC) were identified. Bioinformatic analyses including $\mathrm{GO}$ analysis, pathway analysis, and pathway enrichment analysis was performed to analyse the key metabolic pathways that underlie the thermal tolerance mechanism through protein networks. Quantitative real-time PCR and western blot were performed to validate selected proteins.
\end{abstract}

Results: In the present study, 1223 DEPs were identified under high temperature compared with the control, which included 790 up-regulated and 433 down-regulated proteins. The high-temperature stimulus mainly induced the expression of glutathione S-transferase, heat shock protein, ascorbate peroxidase, manganese superoxide dismutase, ubiquitin-related protein, IhcSR, rubisco activase, serine/threonine protein kinase 2, adenylate kinase, $\mathrm{Ca}^{2+}$-dependent protein kinase (CDPK), disease resistance protein EDS1, metacaspase type II, NDPK2a, 265 proteasome regulatory subunit, ubiquinone oxidoreductase, ATP synthase subunit, SnRK2s, and cytochrome P450. The down-regulated proteins were photosynthesis-related proteins, glutathione reductase, catalase-peroxidase, thioredoxin, thioredoxin peroxidase, PP2C, and carbon fixation-related proteins. Furthermore, biological index analysis indicated that protein content and SOD activity decreased; the value of FV/Fm dropped to the lowest point after culture for $96 \mathrm{~h}$. However, APX activity and MDA content increased under high temperature.

Conclusion: The present study implied an increase in proteins that were associated with the stress response, oxidative phosphorylation, the cytokinin signal transduction pathway, the abscisic acid signal transduction pathway, and the glutathione metabolism pathway. Proteins that were associated with photosynthesis, carbon fixation in photosynthesis organisms, and the photosynthesis antenna protein pathway were decreased. These pathways played a pivotal role in high temperature regulation. These novel proteins provide a good starting point for further research into their functions using genetic or other approaches. These findings significantly improve the understanding of the molecular mechanisms involved in the tolerance of algae to high-temperature stress.

Keywords: U/va prolifera, Proteome analysis, High temperature, iTRAQ

\footnotetext{
* Correspondence: dinger503@163.com; xunianjun@nbu.edu.cn

${ }^{1}$ Marine Sciences and Technology College, Zhejiang Ocean University, Zhoushan, Zhejiang 316000, China

${ }^{2}$ Key Laboratory of Marine Biotechnology of Zhejiang Province, School of

Marine Sciences, Ningbo University, Ningbo, Zhejiang 315211, China
}

(c) The Author(s). 2018 Open Access This article is distributed under the terms of the Creative Commons Attribution 4.0 International License (http://creativecommons.org/licenses/by/4.0/), which permits unrestricted use, distribution, and reproduction in any medium, provided you give appropriate credit to the original author(s) and the source, provide a link to the Creative Commons license, and indicate if changes were made. The Creative Commons Public Domain Dedication waiver (http://creativecommons.org/publicdomain/zero/1.0/) applies to the data made available in this article, unless otherwise stated. 


\section{Background}

Ulva prolifera (Chlorophyta) belongs to Chlorophyta, Ulvales, Ulvaceae and is a type of adaptable large algae. It is rich in protein, carbohydrates, crude fibre, vitamins, amino acids, unsaturated fatty acids and other bioactive substances. $U$. prolifera was developed as a food material, for refining raw materials, and as aquaculture feed. The natural reproductive ability of $U$. prolifera is strong, and it is the dominant species in the Yellow Sea and East China Sea. The breakout of $U$. prolifera in Qingdao since June 2008 has destroyed the natural environment and has a great influence on tourism and the aquatic industry. In addition, $U$. prolifera had been abundant in Xiangshan of the East China Sea from January to March. However, it disappeared in April. Therefore, change in the environment, especially temperature, had a great influence on the growth of $U$. prolifera. To adapt to these environmental changes, $U$. prolifera has developed mechanisms to adapt to different types of stresses including high temperatures, cold, hypersalinity and ultraviolet radiation [1, 2]. High temperature is a major environmental factor that limits $U$. prolifera yield. It can affect photosynthesis, respiration, water balance, membrane stability, hormone levels, and primary and secondary metabolites [3]. Direct injuries that are associated with high temperature include protein denaturation, aggregation, and increased fluidity of membrane lipids. Indirect or slower heat injuries include inactivation of enzymes in chloroplasts and mitochondria, inhibition of protein synthesis, protein degradation and loss of membrane integrity [4-6]. Photosynthesis is a process that is very sensitive to heat stress. The inhibition of photosystem II (PSII) leads to an invariable change in chlorophyll fluorescence [7]. To counter the effects of heat stress on cellular metabolism, plants respond to temperature changes by reprogramming their transcriptome, proteome, metabolome and lipidome. Such changes are aimed at establishing a new steady-state balance of metabolic processes that can enable the organism to function, survive and even reproduce at a higher temperature [5]. Previous studies have focused on physiological changes including photosynthesis, respiration, cell-membrane stability, hormone changes and induced antioxidant systems and heat-shock-protein expression in $U$. prolifera at high temperatures [8-10]. The effect of heat stress on $U$. prolifera appears to be associated with multiple processes and mechanisms including stress-related genes, transcription factors, and metabolism [11]. However, the transcription patterns are not always directly consistent with protein expression levels. To the best of our knowledge, the transcriptome expression analysis of the responses of $U$. prolifera to abiotic stresses such as low light or low temperature have been reported [1]. However, little attention has been given to the proteomic changed analysis of the response of $U$. prolifera to high temperatures.

Recent advances in the use of iTRAQ labelling of peptides and proteins for the relative quantification of an entire organism or cell proteome under different experimental conditions offer promising tools for the discovery of biomarkers at the protein level. Proteomic responses to heat stress have been widely studied in many species, such as Aspergillus flavus [12], Phaeodactylum tricornutum [13], and Pyropia yezoensis [14]. In addition, proteomic analysis also has been used to identify the metabolic responses of Synechocystis PCC 6803 to biofuel butanol, ethanol and hexane treatments [15-17]. However, it has been rarely reported in $U$. prolifera.

We describe here a procedure for proteomic analysis of the $U$. prolifera using iTRAQ labelling to identify differentially expressed proteins upon exposure to high temperature. The goal of the study is to use the molecular information in $U$. prolifera and provide a better understanding of the thermotolerance mechanism in $U$. prolifera and other macroalgae of the same habitats.

\section{Methods \\ Macroalgae collection and preparation}

Macroalgae used for this study were collected from the intertidal zone of the Xiangshan port of the East China Sea, Ningbo, China (121.82424 E, 29.552086 N) during low tide. U. prolifera samples were extensively rinsed with filtered $(0.2 \mu \mathrm{m})$ seawater and placed into an enamel tray to further remove debris and epiphytes. Furthermore, the $U$. prolifera samples were disinfected with $0.2 \% \mathrm{KI}$ for $10 \mathrm{~min}$ and flushed with sterile seawater. Next, $U$. prolifera samples that were inoculated in Provasoli medium [18] with $200 \mu \mathrm{g} / \mathrm{mL}$ ampicillin were placed in a biochemical incubator that was set to the appropriate temperature $\left(25^{\circ} \mathrm{C}\right)$ and light intensity $\left(100 \mu \mathrm{mol} \mathrm{s}{ }^{-}\right.$ ${ }^{1} \mathrm{~m}^{-2}$ on a $12 \mathrm{~h}: 12 \mathrm{~h}$ light dark cycle). To obtain relatively sterile materials, the $\mathcal{U}$. prolifera samples were disinfected and transferred to fresh Provasoli medium every 7 days and continuously cultured for 10 days at $25^{\circ} \mathrm{C}$.

\section{Experimental design}

$U$. prolifera samples were transferred to a hightemperature $35{ }^{\circ} \mathrm{C}$ growth chamber and subjected to the same irradiance and photoperiod (denoted UpHT) as the treatment group, with three biological repeats. In addition, the $U$. prolifera samples were cultured at $25{ }^{\circ} \mathrm{C}$ as the control group (denoted $\mathrm{UpC}$ ), with three biological repeats. The gametophyte thalli were harvested at $3 \mathrm{~h}$, snap frozen using liquid nitrogen and maintained at $-70{ }^{\circ} \mathrm{C}$ until protein extraction. In addition, $U$. prolifera were collected at $3 \mathrm{~h}, 12 \mathrm{~h}, 24 \mathrm{~h}, 48 \mathrm{~h}$ and $96 \mathrm{~h}$ for the determination of physiological indexes. 


\section{Protein extraction}

Proteins were extracted using the following method: the $U$. prolifera samples were disrupted in lysis buffer (including $0.1 \mathrm{M} / \mathrm{L}$ Tris- $\mathrm{HCl}, 1.4 \mathrm{M} / \mathrm{L} \mathrm{NaCl}, 0.02 \mathrm{M} / \mathrm{L}$ $\mathrm{Na}_{2}$ EDTA, 2\% CTAB, 0.1\% DIECA, 2\% PVP K-30, 0.2\% $\beta$-mercaptoethanol with $\mathrm{pH}$ adjusted to 8.0) with enzyme inhibitors (phenylmethyl sulphonyl fluoride, PMSF) using a tissue grinder and were sonicated on ice. The expected proteins were extracted after centrifugation at 25,000 g for 20 mins. The supernatants were carefully removed and mixed with an appropriate volume of cold acetone and stored at $-20{ }^{\circ} \mathrm{C}$ overnight. The mixture was centrifuged again. The pellets were dissolved using lysis buffer. Next, 10 mM DTT (dithiothreitol) was added to the solution, and the solution was maintained at $56{ }^{\circ} \mathrm{C}$ for $1 \mathrm{~h}$ to reduce the disulphide bonds of peptides. Next, $55 \mathrm{mM}$ IAM (iodacetamide) was added to the solution, and the solution was maintained in a darkroom for $45 \mathrm{~min}$. An appropriate volume of chilled acetone was added into the solution, which was maintained at $-20{ }^{\circ} \mathrm{C}$ for $2 \mathrm{~h}$. The pellet was dissolved with lysis buffer to obtain the protein solution. Final protein concentrations were determined using BCA kits according to the manufacturer's instructions.

\section{Protein digestion and ITRAQ labelling}

Each $100 \mu \mathrm{g}$ of protein was digested in trypsin solution (1:10) and incubated at $37{ }^{\circ} \mathrm{C}$ for $4 \mathrm{~h}$. The digested peptides were labelled using iTRAQ reagents according to the manufacturer's instructions. iTRAQ Reagent 6-Plex kit (Applied Biosystems, Foster City, CA, USA) was used for iTRAQ labelling according to the manufacturer's protocol with some modifications. The iTRAQ labelling reagents 113,114 and 115 were used to label three biological replicates from the control group; similarly, 116, 117 and 118 were used to label three biological replicates from UpHT.

\section{Peptide fractionation via reverse phase chromatography}

Prominence high-performance liquid chromatography (HPLC) system (LC-20AB; Shimadzu, Kyoto, Japan) that was connected to a reversed-phase column was used to perform the first dimension of peptide separation. The iTRAQ-labelled sample was dried using a speedvac and resuspended in $2 \mathrm{~mL}$ of $5 \%$ acetonitrile, and the $\mathrm{pH}$ was adjusted to approximately $\mathrm{pH}$ 9.8. The resuspended sample solution was injected into a Gemini C18 column ( $4.6 \times 250 \mathrm{~mm}, 5-\mu \mathrm{m}$ particles). The following separation gradient with buffer B ( $98 \% \mathrm{ACN}, 0.1 \% \mathrm{FA})$ was used at a flow rate of $1000 \mu \mathrm{L} / \mathrm{min}$ : $5 \%$ of mobile phase $B$ in $10 \mathrm{~min}, 5$ to $35 \%$ of mobile phase B in $40 \mathrm{~min}$, and 35 to $95 \%$ of mobile phase B in $1 \mathrm{~min}$. The system was then maintained in $95 \%$ of mobile phase B in 3 min, which was decreased to $5 \%$ within 1 min before equilibrating with $5 \%$ solvent $\mathrm{B}$ for $10 \mathrm{~min}$. Elution was monitored by measuring the absorbance at $214 \mathrm{~nm}$, and fractions are collected every $1 \mathrm{~min}$. The peptides were subjected to nanoelectrospray ionization followed by tandem mass spectrometry (MS/MS) in a Q EXACTIVE (Thermo Fisher Scientific, San Jose, CA) that was coupled online to the HPLC.

\section{Identification and quantification of peptides and proteins}

The MS/MS data were searched against a protein sequence database that was derived from the transcriptome database of $U$. prolifera and partion for other green algae proteins using the ProteinPilot ${ }^{\mathrm{tm}}$ software 4.2 (SCIEX) for peptide identification and quantification. The MS/MS spectra obtained were searched using the following user-defined search parameters. The MS/MS spectra were searched against a decoy database to estimate the false discovery rate (FDR) for peptide identification. For iTRAQ studies, 95\% protein confidence level was used as the identification criterion.

\section{Proteomics data processing}

The raw MS/MS data were converted into ".mgf" files using ProteinPilot software (AB Sciex). Mascot version 2.3.0 (Matrix Sciences, London, UK) was used to search against the transcriptome database of $U$. prolifera and partion for other green algae proteins. To identify false positives, raw spectra from the actual database were compared with a generated database of random sequences. Only peptides with significant scores at the $95 \%$ confidence level were considered reliable and used for protein identification. Protein quantitative ratios were weighted and normalized relative to the median ratio in Mascot. Only proteins with significant quantitative ratios between the two treatments $(p<0.05)$ and with fold changes $>1.2$ or $<$ 0.83 were considered differentially expressed. Functional annotation and classification of proteins for the DEPs was conducted using the Blast2GO program (https:// www.blast2go.com/) [19]. KEGG pathway analysis was performed using (http://kobas.cbi.pku.edu.cn/anno_iden.php). The clustering of the heat map was conducted using Mev4.9. Enrichment analysis was conducted using the singular enrichment analysis (SEA) tool in the agriGO toolkit [20]. Subcellular localizations of proteins were determined using TargetP [21].

\section{RNA isolation and qRT-PCR}

The isolated RNA sequencing samples were also used to perform real-time quantitative (qRT-PCR) analysis. Extraction of total RNA from three biological replicates in $\mathrm{UpHT}$ and three biological replicates in UpC was performed using plant RNA Kit (Omega). The total RNA was reverse transcribed to cDNA using TransScript ${ }^{\circ}$ All-in-One First-Strand cDNA Synthesis SuperMix for qPCR (one-step gDNA removal) (Trans) according to 
the manufacturer's protocol. The purity and integrity of the RNA was verified via absorbance measurements at $260 \mathrm{~nm}$ using a Nanodrop ND-1000 spectrophotometer (NanoDrop Technologies Inc., USA) and via electrophoretic separation using a Bioanalyzer and the RNA Nano kit (Agilent Technologies). From the DEGs, 8 related genes were selected to verify the reliability of the transcriptome by real-time fluorescence quantitative PCR. Quantitative real-time PCR was performed on an ABI Quant Studio6 Flex (USA) using SYBR Green PCR kits (Trans, China) according to the manufacturers' instructions. Each cycle of the thermal amplification followed the universal protocol according to the manufacturer's instructions: $94{ }^{\circ} \mathrm{C}$ for $30 \mathrm{~s}$, followed by $40 \mathrm{cy}-$ cles with $15 \mathrm{~s}$ at $94{ }^{\circ} \mathrm{C}$ and $1 \mathrm{~min}$ at $55{ }^{\circ} \mathrm{C}$. For all qRT-PCR results, $\beta$-actin was used as the housekeeping gene, for which sequences of the specific primers have been previously published. The gene-specific primers used to amplify each gene were as follows: $\beta$-actin forward primer was 5'-AGGATGCATACGTTGGTGAA-3', and $\beta$-Actin reverse primer was 5'-TTGTGGTGC CAAATCTTCTC-3' [8].

\section{Western blot}

The protein samples were electrophoresed via $12 \%$ SDS-PAGE and transferred onto nitrocellulose membranes (Millipore, Germany). After blocking with 5\% skim milk, the membranes were incubated with the rabbit polyclonal anti-HSP70 (Agrisera AS0837, Sweden,1: 3000), Rabbit polyclonal Rubisco large subunit,form I and form II antibody (Agrisera AS03037, Sweden 1:10000) at $4{ }^{\circ} \mathrm{C}$ overnight, respectively. Subsequently, the membranes were washed with $\mathrm{PBS}$ five times and incubated with goat anti-rabbit $\operatorname{IgG}(\mathrm{H}+\mathrm{L})$ secondary antibody (Thermo Fisher Scientific, USA) at a ratio of 1:5000 at room temperature for $2 \mathrm{~h}$. Finally, the membranes were developed using an enhanced chemiluminescence kit (Santa Cruz, USA) [22].

\section{PPI network analysis}

The PPI data of $U$. prolifera were downloaded from the STRING database. Each interaction has a combined score, which represents the reliability of the interaction between the proteins. The PPI interactions with a combined score (0: lowest confidence; 1 : highest confidence) larger than 0.4 were used for further network analysis. All differentially expressed proteins were mapped onto the PPI network, and the Cytoscape tool was used to visualize the network.

\section{Physiological and biochemical indicators}

It was proposed that physiological adaptations of $U$. prolifera may enable it to survive the harsh intertidal environment and contribute to subsequent blooms. To investigate the effects of high temperature on antioxidant activities and photosynthesis indexes, the $U$. prolifera samples were quickly frozen using liquid nitrogen and stored at $-80{ }^{\circ} \mathrm{C}$. For antioxidative enzyme extraction, $1.0 \mathrm{~g}$ of $U$. prolifera was homogenized in $5.0 \mathrm{~mL}$ of extraction buffer containing $1 \mathrm{mM}$ EDTA, 0.05\% Triton-X-100, 2\% PVP, and $1 \mathrm{mM}$ ascorbate in $50 \mathrm{mM}$ phosphate buffer, $\mathrm{pH}$ 7.8. This mixture was centrifuged at $12,000 \mathrm{~g}$ for $20 \mathrm{~min}$ at $4{ }^{\circ} \mathrm{C}[11,23]$. The resulting supernatant was stored at $-20{ }^{\circ} \mathrm{C}$ for the assay of the following antioxidant enzymes.

\section{Superoxide dismutase activity determination}

Total superoxide dismutase activity was determined via the inhibition of the photochemical reduction of the chloride nitroblue tetrazolium (NBT) at $560 \mathrm{~nm}$. The enzyme activity was expressed as unit $\mathrm{U} / \mathrm{g}$ fresh weight, and one SOD unit was defined as the quantity required to inhibit the photoreduction rate of NBT by $50 \%$ [24].

\section{Ascorbate peroxidase (APX) activity determination}

Ascorbate peroxidase (APX) activity was determined by measuring the decrease in absorbance of ascorbic acid (AsA) at $290 \mathrm{~nm}$. The reaction mixture contained $2.6 \mathrm{~mL}$ of PBS (pH 7.5, containing $0.1 \mathrm{mmol} / \mathrm{L}$ EDTA and $0.5 \mathrm{mmol} / \mathrm{L} \mathrm{AsA}), 0.1 \mathrm{~mL}$ of sample, and $0.3 \mathrm{~mL}$ of $2 \mathrm{mmol} / \mathrm{L} \mathrm{H}_{2} \mathrm{O}_{2}$ [24, 25].

\section{Estimation of lipid peroxidation (MDA content)}

Fresh samples (500 $\mathrm{mg}$ each) were homogenized in $10 \mathrm{~mL}$ of $0.1 \%$ trichloroacetic acid (TCA). The homogenate was centrifuged at $15,000 \mathrm{~g}$ for $5 \mathrm{~min}$. Next, $2 \mathrm{~mL}$ aliquot of supernatant was taken and $4 \mathrm{~mL}$ of $0.5 \%$ thiobarbituric acid (TBA) in 20\% TCA was added to it. The mixture was heated at $95{ }^{\circ} \mathrm{C}$ for $30 \mathrm{~min}$ and quickly cooled in an ice bath. After centrifugation at 10,000 g for $10 \mathrm{~min}$ to remove the suspended turbidity, the absorbance of the supernatant was recorded at $532 \mathrm{~nm}$ absorbance on a UV-visible spectrophotometer (Chemito Spectrascan, UV 2600). The value of non-specific absorption at $600 \mathrm{~nm}$ was subtracted. The MDA content was calculated using its absorption coefficient of $155 \mathrm{mmol} / \mathrm{cm}[26,27]$.

\section{Chlorophyll fluorescence parameters measurements}

Chlorophyll fluorescence parameters of the maximum photochemical efficiency $(\mathrm{Fv} / \mathrm{Fm})$ were measured using Water-PAM. Detection of chlorophyll fluorescence was made using a pulse modulation fluorometer (JUNIORPAM, Walz, Germany). At least three algal samples were used for each measurement of chlorophyll fluorescence, and the algae were acclimated to darkness for $10 \mathrm{~min}$ before being analysed. The maximum quantum yield of the photosystem (PS) II of $U$. prolifera was estimated as 
FV/Fm; the photochemical quenching coefficient (qP) and nonphotochemical quenching coefficient (NPQ) were also determined. The rapid light curves (RLCs) consisted of the fluorescence response to eight different and increasing actinic irradiance levels over the range of $0 \sim 820 \mu \mathrm{mol}$ photons $\mathrm{m}^{-2} \mathrm{~s}^{-1}$. The parameters of the RLCs were calculated following the formula described by Jassby and Platt: rETR (relative electron transport rate) $=\mathrm{rETRmax} \times \tan h(\alpha \times I / \mathrm{rETRmax})$, where $\mathrm{rETRmax}$ is the saturated maximum rETR, $\tan h$ is the hyperbolic tangent function, $\alpha$ is the initial slope of the RLC (the efficiency of the electron transport), and $I$ is the incident irradiance [28].

\section{Results and discussion}

\section{Annotation of proteome data}

Protein response to heat stress in $U$. prolifera were revealed by iTRAQ analysis. A total of 283,344 spectra were generated; 15,546 peptides and 4449 proteins were identified with the cut-off of Mascot Percolator Q value $<=0.01$ [29]. Differentially expressed proteins (DEPs) were identified upon an expression ratio $>1.20$ or $<0.83$ and $p<0.05$ under UpHT compared to UpC [30-32]. During high-temperature stress, a total of 1223 proteins were found to be significantly changed, of which, 790 DEPs were up-regulated and 433 DEPs were down-regulated (Fig. 1, Table 1), which indicated that almost $27.50 \%$ of the identified proteins changed their abundance significantly in response to the heat stress.

\section{GO function analysis of DEPs}

GO analysis was conducted on DEPs using the Blast2GO program; 469, 481 and 473 DEPs were successfully assigned to the biological processes, molecular function, and cellular components GO categories, respectively. The distribution of GO terms (the second level) for the three categories is shown in Table 2. For the biological process category, the most abundant groups were metabolic process $(42.20 \%)$, cellular process $(39.40 \%)$, and translation (24.90\%). In addition, 126 DEPs belonged to response to stimulus (26.86\%), of which 86 DEPs were up-regulated and $40 \mathrm{DEPs}$ were down-regulated. For the cellular component category, cells (63.59\%), cell parts (63.59\%), and organelles (51.79\%) were the most abundant groups. In the molecular function category, most of the DEPs were classified into catalytic activity (58.07\%) and binding functions (45.70\%); antioxidant activity only accounted for $0.72 \%$ of the DEPs (Table 2). Furthermore, subcellular localization of the 790 up-regulated characterized proteins showed that 54 proteins $(6.84 \%)$ were located in the chloroplast, 139 proteins $(17.60 \%)$ were assigned to the mitochondria, 42 proteins (5.32\%) belonged to the secretory pathway, and 512 proteins (12\%) were classified as belonging to other locations.

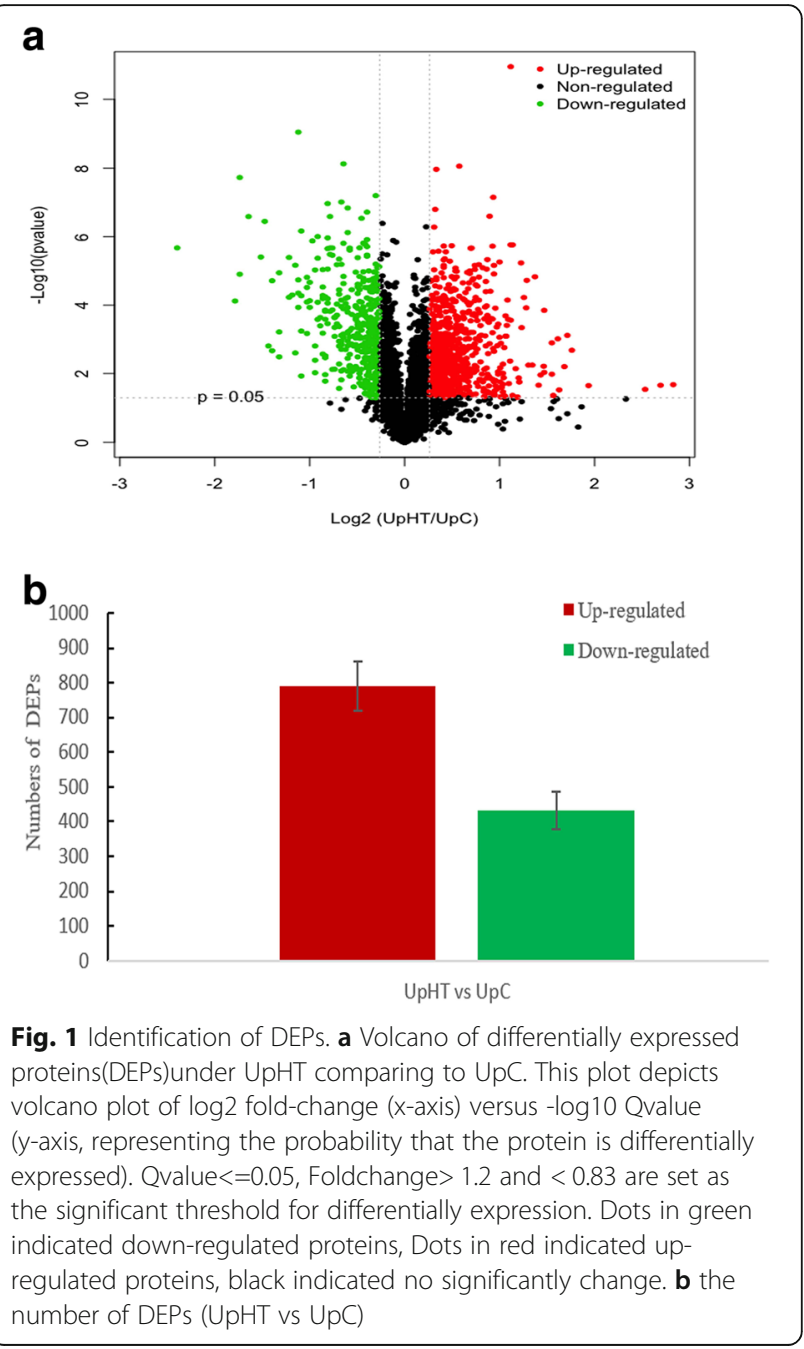

Forty-three of the DEPs had unknown locations (Fig. 2). Furthermore, subcellular localization analysis of the 433 down-regulated proteins showed that quite many chloroplast proteins and mitochondria proteins are related to the thermotolerance of $U$. prolifera.

\section{COG function class of DEPs}

In the COG function analysis, the largest category was "translation, ribosomal structure and biogenesis" (161 DEPs up-regulated, 13 DEPs down-regulated), followed by "posttranslational modification, protein turnover, molecular chaperone" (79DEPs up-regulated and 20 DEPs down-regulated) and "general function prediction only" (39 DEPs up-regulated and 24 DEPs down-regulated) (Fig. 3).

\section{KEGG pathway analysis of DEPs}

KEGG pathway analysis of DEPs were conducted online at http://www.genome.jp/kegg/kegg2.html; 557 up-regulated proteins were mapped to 97 KEGG pathways, and the top 15 pathways are listed in Table 3 . The pathway enrichment 
Table 1 Partion DEPs under UpHT vs UpC conditions

\begin{tabular}{|c|c|c|c|c|}
\hline & Protein_ID & Description & AccessionNo. & GO annotation \\
\hline \multicolumn{5}{|c|}{$\begin{array}{l}\text { Up- } \\
\text { regulated }\end{array}$} \\
\hline 454 & CL232_Contig1_All & $\begin{array}{l}\text { glutathione S-transferase [Chlorella } \\
\text { variabilis] }\end{array}$ & gi|307,109,344|gb|EFN57582.1| & antioxidant activity \\
\hline 1426 & Unigene12106_All & $\begin{array}{l}\text { glutathione S-transferase [Coccomyxa } \\
\text { subellipsoidea C-169] }\end{array}$ & gi|384,253,101|gb|ElE26576.1| & catalytic_activity \\
\hline 1483 & CL2198_Contig2_All & $\begin{array}{l}\text { glutathione S-transferase [Coccomyxa } \\
\text { subellipsoidea C-169] }\end{array}$ & gi|384,253,101|gb|ElE26576.1| & catalytic_activity \\
\hline 695 & Unigene6725_All & $\begin{array}{l}\text { minus strand polyubiquitin } \\
\text { [Aureococcus anophagefferens] }\end{array}$ & gi|323,454,622|gb|EGB10492.1| & antioxidant activity \\
\hline 2681 & CL6691_Contig2_All & MnSOD [U. prolifera] & gi|149,275,665|gb|ABR23158.1| & antioxidant activity \\
\hline 202 & Unigene40313_All & $\begin{array}{l}\text { heat shock protein 90, cytosolic } \\
\text { [Ostreococcus lucimarinus CCE9901] }\end{array}$ & gi|166,770|gb|AAA32822.1| & binding \\
\hline 984 & gi|297,592,060|gb|AD|46845_1| & $\begin{array}{l}\text { heat shock protein 70B [Volvox carteri } \\
f_{\text {_ nagariensis] }}\end{array}$ & gi|302,853,561|ref.|XP_002958295.1| & binding \\
\hline 2451 & CL5666_Contig1_All & heat shock protein 90 [U/va pertusa] & gi|371,940,442|dbj|BAL45643.1| & binding \\
\hline 2543 & CL2081_Contig1_All & $\begin{array}{l}\text { heat shock protein } 90 \mathrm{C} \\
\text { [Chlamydomonas reinhardti] }\end{array}$ & gi|159,490,014|ref.|XP_001702984.1| & binding \\
\hline 2944 & gi|300,269,068|gb|EFJ53248_1| & $\begin{array}{l}\text { heat shock protein } \mathrm{Hsp} 70 \mathrm{H} \text { [Volvox } \\
\text { carteri f_nagariensis] }\end{array}$ & gi|302,829,372|ref.|XP_002946253.1| & binding \\
\hline 3056 & gi|158,270,891|gb|EDO96722_1| & $\begin{array}{l}\text { heat shock protein } 90 \mathrm{C} \\
\text { [Chlamydomonas reinhardti] }\end{array}$ & gi|159,490,014|ref.|XP_001702984.1| & binding \\
\hline 3197 & gi|254,558,246|gb|ACT67905_1| & heat shock protein 70 [Ulva pertusa] & gi|254,558,246|gb|ACT67905.1| & binding \\
\hline 4221 & CL413_Contig1_All & $\begin{array}{l}\text { minus strand heat shock protein } 60 \\
\text { [Ulva pertusa] }\end{array}$ & gi|371,940,440|dbj|BAL45642.1| & binding \\
\hline 75 & CL152_Contig5_All & $\begin{array}{l}\text { ubiquitin-60S ribosomal protein } L 40 \\
\text { [Vitis vinifera] }\end{array}$ & gi|359,482,015|ref.|XP_002282071.2| & structural_molecule_activity \\
\hline 2429 & Unigene16018_All & $\begin{array}{l}\text { NADH:ubiquinone oxidoreductase } 11 \\
\text { kDa subunit [Chlamydomonas reinhardti] }\end{array}$ & gi|159,475,537|ref.|XP_001695875.1| & catalytic_activity \\
\hline 2470 & gi|1,001,185,371|gb|AML80572_1| & $\begin{array}{l}\text { NADH-ubiquinone oxidoreductase } 49 \\
\text { kDa subunit (mitochondrion) [U. } \\
\text { prolifera] }\end{array}$ & gi|49,147,198|ref.|YP_025791.1| & binding catalytic_activity \\
\hline 2717 & Unigene42071_All & $\begin{array}{l}\text { similar to ubiquitin conjugating } \\
\text { enzyme E2 [Cyanidioschyzon merolae } \\
\text { strain 10D] }\end{array}$ & gi|449,018,406|dbj|BAM81808.1| & binding catalytic_activity \\
\hline 4135 & CL4653_Contig2_All & $\begin{array}{l}\text { peptidase } C 12 \text {, ubiquitin carboxyl- } \\
\text { terminal hydrolase } 1 \text { [Coccomyxa } \\
\text { subellipsoidea C-169] }\end{array}$ & gi|384,249,810|gb|EIE23291.1| & cell cell_part \\
\hline 3660 & CL649_Contig6_All & $\begin{array}{l}\text { Rubisco activase [Coccomyxa } \\
\text { subellipsoidea C-169] }\end{array}$ & gi|384,253,108|gb|ElE26583.1| & binding \\
\hline 1212 & CL492_Contig1_All & $\begin{array}{l}\text { bifunctional sensory photoreceptor } \\
\text { [Volvox carteri f_ nagariensis] }\end{array}$ & gi|302,844,634|ref.|XP_002953857.1| & Energy convert \\
\hline 622 & gi|315,319,013|gb|ADU04518_1| & LhcSR [U. prolifera] & gi|315,319,013|gb|ADU04518.1| & Energy convert \\
\hline 621 & gi|158,277,339|gb|EDP03108_1| & $\begin{array}{l}\text { serine/threonine protein kinase } \\
2 \text { [Chlamydomonas reinhardtii] }\end{array}$ & gi|159,472,056|ref.|XP_001694172.1| & Signal \\
\hline 2807 & Unigene25558_All & $\begin{array}{l}\text { calcium-dependent protein kinase } \\
\text { [Chlamydomonas reinhardti] }\end{array}$ & gi|159,464,829|ref.|XP_001690644.1| & Signal \\
\hline 329 & CL2614_Contig3_All & $\begin{array}{l}\text { ArfB-family small GTPase [Coccomyxa } \\
\text { subellipsoidea C-169] }\end{array}$ & gi|384,244,739|gb|EIE18237.1| & Signal \\
\hline 1431 & Unigene44820_All & $\begin{array}{l}\text { ras-related gtp-binding protein } \\
\text { [Micromonas sp_ RCC299] }\end{array}$ & gi|255,075,683|ref.|XP_002501516.1| & Signal \\
\hline 2089 & Unigene4025_All & $\begin{array}{l}\text { minus strand ras-related protein } \\
\text { Rab2BV [Coccomyxa subellipsoidea } \\
\text { C-169] }\end{array}$ & gi|307,102,647|gb|EFN50917.1| & Signal \\
\hline
\end{tabular}


Table 1 Partion DEPs under UpHT vs UpC conditions (Continued)

\begin{tabular}{|c|c|c|c|c|}
\hline & Protein_ID & Description & AccessionNo. & GO annotation \\
\hline 3066 & gi|158,271,373|gb|EDO97193_1| & $\begin{array}{l}\text { ARF-like GTPase } \\
\text { [Chlamydomonas reinhardtii] }\end{array}$ & gi|159,488,198|ref.|XP_001702104.1| & Signal \\
\hline 63 & Unigene7624_All & $\begin{array}{l}\text { NDPK2a nucleotide diphosphate } \\
\text { kinase } 2 \text { protein [Physcomitrella } \\
\text { patens subsp_patens] }\end{array}$ & gi|168,020,398|ref.|XP_001762730.1| & Signal \\
\hline 3025 & CL5370_Contig1_All & $\begin{array}{l}\text { adenylate kinase [Coccomyxa } \\
\text { subellipsoidea C-169] }\end{array}$ & gi|384,247,647|gb|EIE21133.1| & Signal \\
\hline 1512 & CL3463_Contig1_All & $\begin{array}{l}\text { calcium-dependent protein } \\
\text { kinase(CDPK)[Coccomyxa } \\
\text { subellipsoidea C-169] }\end{array}$ & gi|384,245,250|gb|EIE18745.1| & Signal \\
\hline 3467 & CL3910_Contig4_All & $\begin{array}{l}\text { calcium-dependent protein kinase } \\
\text { (CDPK)[Volvox carteri f_ nagariensis] }\end{array}$ & gi|384,245,250|gb|EIE18745.1| & Signal \\
\hline 4337 & Unigene26962_All & $\begin{array}{l}\text { calcium-dependent protein kinase } \\
\text { (CDPK) [Chlamydomonas reinhardti] }\end{array}$ & gi|159,464,829|ref.|XP_001690644.1| & Signal \\
\hline 3383 & CL7329_Contig1_All & $\begin{array}{l}\text { histidine-aspartic acid } \\
\text { phosphotransferase [Chlamydomonas } \\
\text { reinhardti] }\end{array}$ & gi|159,463,834|ref.|XP_001690147.1| & Signal \\
\hline 1545 & CL4735_Contig1_All & $\begin{array}{l}\text { kinase-like protein-SnRK2 [Coccomyxa } \\
\text { subellipsoidea C-169] }\end{array}$ & gi|384,245,989|gb|EIE19481.1| & Signal \\
\hline 4259 & CL6485_Contig1_All & $\begin{array}{l}\text { metacaspase type II [Chlamydomonas } \\
\text { reinhardtii] }\end{array}$ & gi|159,467,293|ref.|XP_001691826.1| & catalytic_activity \\
\hline 1524 & gi|28,076,780|gb|AAO31578_1| & $\begin{array}{l}\text { ribulose-1,5-bisphosphate carboxylase/ } \\
\text { oxygenase large subunit, partial } \\
\text { (chloroplast) [Ulva lactuca] }\end{array}$ & gi|28,076,780|gb|AAO31578.1| & $\begin{array}{l}\text { Carbohydrate transport and } \\
\text { metabolism }\end{array}$ \\
\hline 1650 & gi|145,567,455|gb|ABP82085_1| & $\begin{array}{l}\text { ribulose-1,5-bisphosphate carboxylase/ } \\
\text { oxygenase large subunit, partial } \\
\text { (chloroplast) [Ulva sp_WELT A027500] }\end{array}$ & gi|219,932,444|emb|CAR65339.1| & $\begin{array}{l}\text { Carbohydrate transport and } \\
\text { metabolism }\end{array}$ \\
\hline 3021 & gi|158,282,407|gb|EDP08159_1| & triacylglycerol lipase-like protein(EDS1) & gi|159,476,166|ref.|XP_001696182.1| & Plant-pathogen interaction \\
\hline
\end{tabular}

Down-

regulated

$\begin{array}{ll}397 & \text { CL206_Contig2_All } \\ 1080 & \text { Unigene25013_All } \\ 1140 & \text { CL6594_Contig3_All } \\ 1295 & \text { CL4649_Contig1_All } \\ 1603 & \text { Unigene17811_All } \\ 1611 & \text { CL1851_Contig5_All }\end{array}$

1686

gi|158,277,735|gb|EDP03502_1|

1762 CL891_Contig1_All

$1828 \quad$ Unigene18752_All

1855 CL7525_Contig1_All

2532 gi|1,181,548|gb|AAA86855_1|

$2584 \quad$ Unigene27523_All

\section{[Chlamydomonas reinhardti]}

elongation factor 2-like

[Solanum lycopersicum]

methionine sulfoxide reductase B [Ulva fasciata]

oxygen-evolving enhancer protein 1 precursor [Volvox carteri f_nagariensis]

ferritin [Ulva fasciata]

chloroplast stem-loop-binding protein [Chlamydomonas reinhardtii]

TPA_inf: chloroplast light-harvesting complex II protein precursor Lhcbm5 [Acetabularia acetabulum]

Adenylosuccinate synthetase, chloroplastic;

actin [Ulva linza]

putative early light-inducible protein 2 [Ulva linza]

chloroplast glycerolipid omega-3-fatty acid desaturase [Chlamydomonas reinhardti]

Glyceraldehyde-3-phosphate dehydrogenase A, chloroplastic;

minus strand plastocyanin precursor [Ulva pertusa]

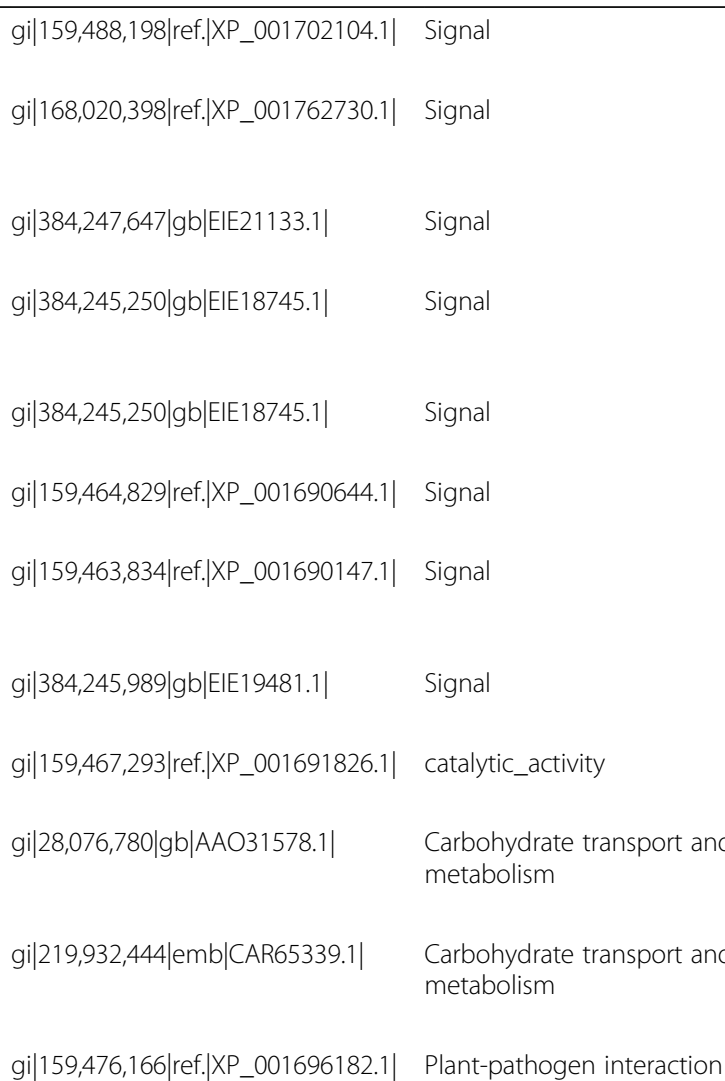


Table 1 Partion DEPs under UpHT vs UpC conditions (Continued)

\begin{tabular}{|c|c|c|c|c|}
\hline & Protein_ID & Description & AccessionNo. & GO annotation \\
\hline 2923 & gi|344,012|dbj|BAA02024_1| & $\begin{array}{l}\text { photosystem II D1 protein (chloroplast) } \\
\text { [Pinus thunbergii] }\end{array}$ & gi|7,524,753|ref.|NP_042347.1| & Response to stimulus \\
\hline 3498 & Unigene37824_All & $\begin{array}{l}\text { minus strand tubulin beta } \\
\text { [Nannochloropsis gaditana CCMP526] }\end{array}$ & gi|422,295,792|gb|EKU23091.1| & Response to stimulus \\
\hline 3524 & gi|158,271,667|gb|EDO97482_1| & $\begin{array}{l}\text { porphobilinogen deaminase } \\
\text { [Chlamydomonas reinhardti] }\end{array}$ & gi|159,486,921|ref.|XP_001701485.1| & Response to stimulus \\
\hline 3644 & CL438_Contig1_All & $\begin{array}{l}\text { fructose-1,6-bisphosphatase } \\
\text { [Chlamydomonas reinhardtii] }\end{array}$ & gi|159,465,323|ref.|XP_001690872.1| & Response to stimulus \\
\hline 3749 & CL930_Contig2_All & $\begin{array}{l}\text { glutamate-1-semialdehyde } \\
\text { aminotransferase [Chlamydomonas } \\
\text { reinhardti] }\end{array}$ & gi|384,247,824|gb|ElE21309.1| & Response to stimulus \\
\hline 3798 & gi|288,816,175|gb|ADC54911_1| & $\begin{array}{l}\text { oxygen-evolving enhancer protein, } \\
\text { partial [Ulva sp_EE2] }\end{array}$ & gi|288,816,175|gb|ADC54911.1| & Response to stimulus \\
\hline 1056 & gi|323,320,892/gb|ADX36432_1| & $\begin{array}{l}\text { ribulose-1,5-bisphosphate carboxylase/ } \\
\text { Oxygenase large subunit, partial } \\
\text { (chloroplast) [U. prolifera] }\end{array}$ & gi|32,492,888|gb|AAP85534.1| & $\begin{array}{l}\text { Carbohydrate transport and } \\
\text { metabolism }\end{array}$ \\
\hline 1767 & gi|145,567,588|gb|ABP82151_1| & $\begin{array}{l}\text { ribulose-1,5-bisphosphate carboxylase/ } \\
\text { oxygenase large subunit, partial } \\
\text { (chloroplast) [Ulva sp_WELT A027397] }\end{array}$ & gi|159,475,703|ref.|XP_001695958.1| & $\begin{array}{l}\text { Carbohydrate transport and } \\
\text { metabolism }\end{array}$ \\
\hline 1854 & Unigene19826_All & $\begin{array}{l}\text { minus strand pyruvate orthophosphate } \\
\text { dikinase [Ulva linza] }\end{array}$ & gi|255,076,895|ref.|XP_002502111.1| & $\begin{array}{l}\text { Carbohydrate transport and } \\
\text { metabolism }\end{array}$ \\
\hline 2044 & CL7324_Contig1_All & $\begin{array}{l}\text { glyceraldehyde-3-phosphate } \\
\text { dehydrogenase, cytosolic-like [Cucumis } \\
\text { sativus] }\end{array}$ & gi|384,245,592|gb|ElE19085.1| & $\begin{array}{l}\text { Carbohydrate transport and } \\
\text { metabolism }\end{array}$ \\
\hline 2532 & gi|1,181,548|gb|AAA86855_1| & $\begin{array}{l}\text { glyceraldehyde-3-phosphate } \\
\text { dehydrogenase A, chloroplastic; }\end{array}$ & gi|323,320,892|gb|ADX36432.1| & $\begin{array}{l}\text { Carbohydrate transport and } \\
\text { metabolism }\end{array}$ \\
\hline 2561 & CL2687_Contig1_All & $\begin{array}{l}\text { minus strand pyruvate orthophosphate } \\
\text { dikinase [U. prolifera] }\end{array}$ & gi|255,076,279|ref.|XP_002501814.1| & $\begin{array}{l}\text { Carbohydrate transport and } \\
\text { metabolism }\end{array}$ \\
\hline 2674 & gi|145,567,931|gb|ABP82322_1| & $\begin{array}{l}\text { ribulose-1,5-bisphosphate carboxylase/ } \\
\text { oxygenase large subunit, partial } \\
\text { (chloroplast) [Ulva sp_WELT A027743] }\end{array}$ & gi|449,475,837|ref.|XP_004154566.1| & $\begin{array}{l}\text { Carbohydrate transport and } \\
\text { metabolism }\end{array}$ \\
\hline 2812 & CL1803_Contig1_All & $\begin{array}{l}\text { phosphoglycerate kinase } \\
\text { [Chlamydomonas reinhardtii] }\end{array}$ & gi|159,463,282|ref.|XP_001689871.1| & $\begin{array}{l}\text { Carbohydrate transport and } \\
\text { metabolism }\end{array}$ \\
\hline 2938 & gi|342,316,037|gb|AEL22116_1| & $\begin{array}{l}\text { pyruvate orthophosphate dikinase } \\
\text { [Ulva linza] }\end{array}$ & gi|378,940,382|gb|AFC75637.1| & $\begin{array}{l}\text { Carbohydrate transport and } \\
\text { metabolism }\end{array}$ \\
\hline 3212 & gi|145,568,009|gb|ABP82361_1| & $\begin{array}{l}\text { ribulose-1,5-bisphosphate carboxylase/ } \\
\text { oxygenase large subunit, partial } \\
\text { (chloroplast) [Ulva sp_WELT A027348] }\end{array}$ & gi|145,568,333|gb|ABP82523.1| & $\begin{array}{l}\text { Carbohydrate transport and } \\
\text { metabolism }\end{array}$ \\
\hline 3644 & CL438_Contig1_All & $\begin{array}{l}\text { fructose-1,6-bisphosphatase } \\
\text { [Chlamydomonas reinhardti] }\end{array}$ & gi|342,316,037|gb|AEL22116.1| & $\begin{array}{l}\text { Carbohydrate transport and } \\
\text { metabolism }\end{array}$ \\
\hline 3884 & CL5964_Contig1_All & $\begin{array}{l}\text { minus strand starch branching enzyme } \\
\text { ॥ [Parachlorella kessleri] }\end{array}$ & gi|194,396,261|gb|ACF60500.1| & $\begin{array}{l}\text { Carbohydrate transport and } \\
\text { metabolism }\end{array}$ \\
\hline 1080 & Unigene25013_All & $\begin{array}{l}\text { methionine sulfoxide reductase } \\
\text { B [Ulva fasciata] }\end{array}$ & gi|197,631,364|gb|ACH70611.1| & $\begin{array}{l}\text { Posttranslational modification, } \\
\text { protein turnover, chaperones }\end{array}$ \\
\hline 2047 & gi|300,259,068|gb|EFJ43299_1| & $\begin{array}{l}\text { molecular chaperone [Volvox carteri } \\
\text { f_ nagariensis] }\end{array}$ & gi|302,848,253|ref.|XP_002955659.1| & $\begin{array}{l}\text { Posttranslational } \\
\text { modification, protein } \\
\text { turnover, chaperones }\end{array}$ \\
\hline 2273 & gi|158,279,996|gb|EDP05755_1| & $\begin{array}{l}\text { signal peptide peptidase, partial } \\
\text { [Chlamydomonas reinhardti] }\end{array}$ & gi|159,464,533|ref.|XP_001690496.1| & $\begin{array}{l}\text { Posttranslational } \\
\text { modification, protein } \\
\text { turnover, chaperones }\end{array}$ \\
\hline 2744 & Unigene5984_All & thioredoxin $\times$ (ISS) [Ostreococcus tauri] & gi|308,804,023|ref.|XP_003079324.1| & $\begin{array}{l}\text { Posttranslational } \\
\text { modification, protein } \\
\text { turnover, chaperones }\end{array}$ \\
\hline 3388 & Unigene9950_All & $\begin{array}{l}\text { thioredoxin dependent peroxidase } \\
\text { [Chlamydomonas reinhardti] }\end{array}$ & gi|159,489,214|ref.|XP_001702592.1| & $\begin{array}{l}\text { Posttranslational modification, } \\
\text { protein turnover, chaperones }\end{array}$ \\
\hline
\end{tabular}


Table 1 Partion DEPs under UpHT vs UpC conditions (Continued)

\begin{tabular}{|c|c|c|c|c|}
\hline & Protein_ID & Description & AccessionNo. & GO annotation \\
\hline 4415 & CL3087_Contig4_All & $\begin{array}{l}\text { molecular chaperone [Volvox carteri } \\
f_{\text {_ nagariensis] }}\end{array}$ & gi|302,839,709|ref.|XP_002951411.1| & $\begin{array}{l}\text { Posttranslational } \\
\text { modification, protein } \\
\text { turnover, chaperones }\end{array}$ \\
\hline 141 & CL451_Contig4_All & $\begin{array}{l}\text { diadenosine tetraphosphatase and } \\
\text { related serine/threonine protein } \\
\text { phosphatases[Ch/orella variabilis] }\end{array}$ & gi|307,103,797|gb|EFN52054.1| & $\begin{array}{l}\text { Signal transduction } \\
\text { mechanisms }\end{array}$ \\
\hline 242 & CL5191_Contig2_All & $\begin{array}{l}\text { serine/threonine protein phosphatase } \\
\text { [Guillardia theta CCMP2712] }\end{array}$ & gi|348,671,120|gb|EGZ10941.1| & $\begin{array}{l}\text { Signal transduction } \\
\text { mechanisms }\end{array}$ \\
\hline 250 & Unigene7524_All & $\begin{array}{l}\text { protein serine/threonine phosphatase } \\
\text { 2C [Coccomyxa subellipsoidea C-169] }\end{array}$ & gi|307,104,872|gb|EFN53124.1| & $\begin{array}{l}\text { Signal transduction } \\
\text { mechanisms }\end{array}$ \\
\hline 3622 & CL6423_Contig1_All & $\begin{array}{l}\text { minus strand protein tyrosine } \\
\text { phosphatase [Ectocarpus siliculosus] }\end{array}$ & gi|299,1 16,693|emb|CBN74838.1| & $\begin{array}{l}\text { Signal transduction } \\
\text { mechanisms }\end{array}$ \\
\hline 4212 & CL4299_Contig1_All & $\begin{array}{l}\text { serine/threonine protein phosphatase } \\
\text { [Volvox carteri __ nagariensis] }\end{array}$ & gi|302,851,958|ref.|XP_002957501.1| & $\begin{array}{l}\text { Signal transduction } \\
\text { mechanisms }\end{array}$ \\
\hline 2947 & Unigene205_All & $\begin{array}{l}\text { phytoene dehydrogenase and related } \\
\text { proteins[Chlorella variabilis] }\end{array}$ & gi|384,249,750|gb|EIE23231.1| & $\begin{array}{l}\text { Secondary metabolites } \\
\text { biosynthesis, transport }\end{array}$ \\
\hline 3019 & gi|158,273,526|gb|EDO99315_1| & $\begin{array}{l}\text { cytochrome P450, CYP85 clan, partial } \\
\text { [Chlamydomonas reinhardti] }\end{array}$ & gi|159,481,496|ref.|XP_001698815.1| & $\begin{array}{l}\text { Secondary metabolites } \\
\text { biosynthesis }\end{array}$ \\
\hline 3998 & CL7806_Contig1_All & $\begin{array}{l}\text { carotenoid cleavage dioxygenase } \\
\text { [Chlamydomonas reinhardti] }\end{array}$ & gi|159,474,908|ref.|XP_001695565.1| & $\begin{array}{l}\text { Secondary metabolites } \\
\text { biosynthesis }\end{array}$ \\
\hline 4156 & CL6885_Contig2_All & $\begin{array}{l}\text { phytoene dehydrogenase and related } \\
\text { proteins [Volvox carteri f_nagariensis] }\end{array}$ & gi|302,835,624|ref.|XP_002949373.1| & $\begin{array}{l}\text { Secondary metabolites } \\
\text { biosynthesis }\end{array}$ \\
\hline 397 & CL206_Contig2_All & $\begin{array}{l}\text { elongation factor 2-like [Solanum } \\
\text { lycopersicum] }\end{array}$ & gi|356,525,774|ref.|XP_003531498.1| & Translation \\
\hline 4324 & gi|452,119,419|gb|AGG09538_1| & $\begin{array}{l}\text { elongation factor Tu, partial } \\
\text { (chloroplast) [U. prolifera] }\end{array}$ & gi|452,119,405|gb|AGG09531.1| & Translation \\
\hline 756 & Unigene5495_All & $\begin{array}{l}\text { oxidoreductase [Coccomyxa } \\
\text { subellipsoidea C-169] }\end{array}$ & gi|384,249,901|gb|ElE23381.1| & $\begin{array}{l}\text { Energy production and } \\
\text { conversion }\end{array}$ \\
\hline 1323 & CL514_Contig1_All & glutathione reductase [Ulva fasciata] & gi|82,658,844|gb|ABB88584.1| & $\begin{array}{l}\text { Energy production and } \\
\text { conversion }\end{array}$ \\
\hline 1554 & CL3939_Contig2_All & glutathione reductase [Ulva fasciata] & gi|82,658,844|gb|ABB88584.1| & $\begin{array}{l}\text { Energy production and } \\
\text { conversion }\end{array}$ \\
\hline 1808 & Unigene43735_All & $\begin{array}{l}\text { photosystem I subunit VII (chloroplast) } \\
\text { [Trebouxiophyceae sp_ MX-AZO1] }\end{array}$ & gi|357,467,753|ref.|XP_003604161.1| & $\begin{array}{l}\text { Energy production and } \\
\text { conversion }\end{array}$ \\
\hline 2584 & Unigene27523_All & plastocyanin precursor [U/va pertusa] & gi|48,526,878|gb|AAT45616.1| & $\begin{array}{l}\text { Energy production and } \\
\text { conversion }\end{array}$ \\
\hline
\end{tabular}

analysis showed that the most significantly enriched pathways among the 557 up-regulated proteins were ribosome $(134,24.05 \%)$, oxidative phosphorylation $(32,5.75 \%)$, proteasome (14, 2.51\%), glutathione metabolism (15, 2.69\%), SNARE interactions in vesicular transport $(5,0.89 \%)$ and linoleic acid metabolism (3, 0.53\%) (Fig. 4a). Two hundred and thirty-three down-regulated proteins were enriched in photosynthesis $(15,6.44 \%)$, carbon fixation in photosynthesis organisms $(15,6.44 \%)$, photosynthesis antenna proteins $(8,8.43 \%)$, metabolic pathways $(91,39.06 \%)$ and lysine biosynthesis (3, 1.2\%) (Fig. 4b). At present, glutathione S-transferase, heat shock proteins, MnSOD and ubiquitin-related proteins were up-regulated. The results indicated that upon high-temperature stimulus, $U$. prolifera induces defence mechanisms, and the expression of heat shock proteins and antioxidant-associated proteins is increased.

\section{Expression analysis of DEPs in response to stimulus}

Abiotic stresses such as high temperature, low temperature, and drought induced plant defence mechanisms, including the expression of antioxidant enzymes to regulate their adaptation [33]. In higher plants, the signal response to high-temperature stress involves a reduction in the synthesis of normal proteins and is accompanied by accelerated expression of heat-responsive 
Table 2 GO analysis of DEPs (UpHT vs UpC) (top15)

\begin{tabular}{|c|c|c|c|}
\hline Gene Ontology term & Cluster frequency & Protein frequency of use & $P$-value \\
\hline translation & 117 out of 469 genes, $24.9 \%$ & 192 out of 1588 genes, $12.1 \%$ & $3.420412 \mathrm{e}-22$ \\
\hline protein metabolic process & 198 out of 469 genes, $42.2 \%$ & 424 out of 1588 genes, $26.7 \%$ & $7.955088 \mathrm{e}-19$ \\
\hline cellular protein metabolic process & 185 out of 469 genes, $39.4 \%$ & 389 out of 1588 genes, $24.5 \%$ & $2.034726 \mathrm{e}-18$ \\
\hline gene expression & 131 out of 469 genes, $27.9 \%$ & 251 out of 1588 genes, $15.8 \%$ & $1.381491 \mathrm{e}-16$ \\
\hline cellular macromolecule biosynthetic process & 136 out of 469 genes, $29.0 \%$ & 275 out of 1588 genes, $17.3 \%$ & $1.195581 \mathrm{e}-14$ \\
\hline response to stimulus & 126 out of 469 genes, $26.86 \%$ & 465 of 1588 in all the Protein & $1.921323 e-6$ \\
\hline structural constituent of ribosome & 93 out of 481 genes, $19.3 \%$ & 114 out of 1743 genes, $6.5 \%$ & $1.412589 \mathrm{e}-35$ \\
\hline structural molecule activity & 104 out of 481 genes, $21.6 \%$ & 142 out of 1743 genes, $8.1 \%$ & $2.312654 \mathrm{e}-32$ \\
\hline rRNA binding & 31 out of 481 genes, $6.4 \%$ & 33 out of 1743 genes, $1.9 \%$ & $6.624359 \mathrm{e}-16$ \\
\hline RNA binding & 73 out of 481 genes, $15.2 \%$ & 131 out of 1743 genes, $7.5 \%$ & $1.526571 \mathrm{e}-12$ \\
\hline nucleic acid binding & 98 out of 481 genes, $20.4 \%$ & 224 out of 1743 genes, $12.9 \%$ & $1.810790 \mathrm{e}-08$ \\
\hline ribonucleoprotein complex & 138 out of 473 genes, $29.2 \%$ & 187 out of 1477 genes, $12.7 \%$ & $1.942048 \mathrm{e}-36$ \\
\hline ribosome & 122 out of 473 genes, $25.8 \%$ & 166 out of 1477 genes, $11.2 \%$ & $1.634089 e-31$ \\
\hline non-membrane-bounded organelle & 165 out of 473 genes, $34.9 \%$ & 281 out of 1477 genes, $19.0 \%$ & $4.775761 \mathrm{e}-25$ \\
\hline intracellular non-membrane-bounded organelle & 165 out of 473 genes, $34.9 \%$ & 281 out of 1477 genes, $19.0 \%$ & $4.775761 \mathrm{e}-25$ \\
\hline macromolecular complex & 214 out of 473 genes, $45.2 \%$ & 409 out of 1477 genes, $27.7 \%$ & $3.124677 \mathrm{e}-24$ \\
\hline
\end{tabular}

genes and HSPs. Reactive oxygen species (ROS) refer to free radicals, including hydrogen peroxide $\left(\mathrm{H}_{2} \mathrm{O}_{2}\right)$, singlet oxygen $\left(\mathrm{O}_{2}{ }^{-}\right)$, and hydroxyl radicals $\left(\mathrm{OH}^{-}\right)$. Excessive amounts of ROS can damage macromolecules and cell membranes. To remove toxic ROS, algae have developed a number of antioxidant systems that serve as protective mechanisms, namely, superoxide dismutases (SOD), catalases, peroxidases, thioredoxin, and glutathione [34]. In the present study, high-temperature stress can lead to protein misfolding and inactivation and desiccation of plant cells. Heat shock proteins are important molecular chaperones of the Hsp family, which prevents the aggregation of misfolded proteins. Significant up-regulation was observed for the heat shock protein family, which included 90 (CL5666_Contig1_All, 1.5-fold), 90C (Unigene40313_All, 1.78 -fold), 70 (gi|219764948|emb|CAW63927_1|, 1.4-fold), ClpB chaperone, Hsp100 family (CL517_Contig2_All, 1.64-fold). Therefore, it could play a role in protecting the protein structure early in the stress response. DNA repair protein Rad50 (CL6208_Contig1_All, 1.22-fold) were identified and significantly up-regulated under high temperature. It means that the DNA repair protein Rad50 played an important role in DNA damage induced by high temperature. We also determined that antioxidant proteins exhibited highly dynamic changes in response to the high-temperature stimulus. Reactive oxygen or nitrogen species are generated in the plant cell during the extreme stress condition,
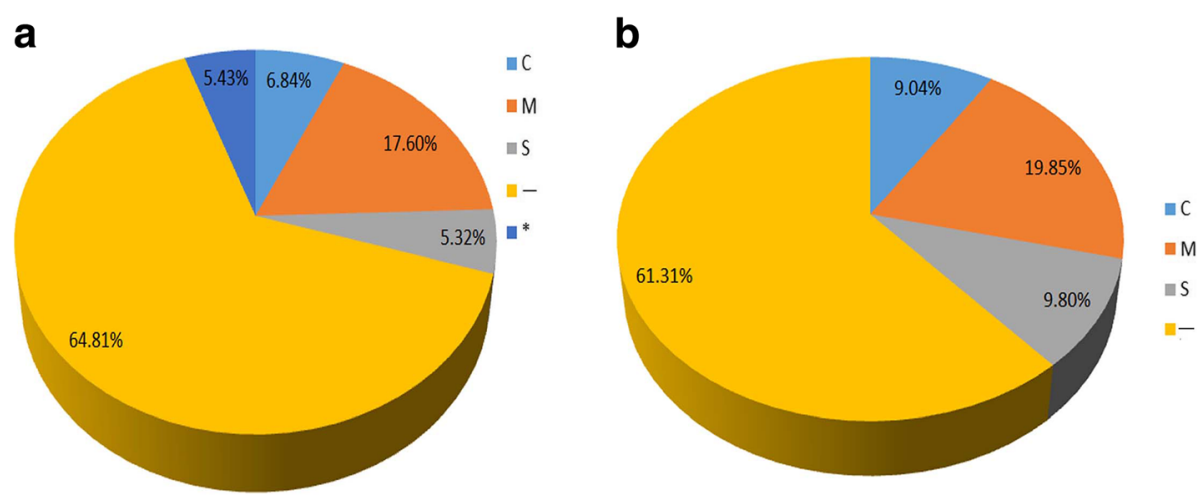

Fig. 2 Subcellular localization of the differentiallyexpressed proteins under heat stress. a Subcellular localization of the up-regulated proteins $\mathbf{b}$ Subcellular localization of the down-regulated proteins Note: C: Chloroplast, i.e. the sequence contains CTP, a chloroplasttransit peptide; M: Mitochondrion, i.e. the sequence contains MTP, amitochondrial targeting peptide; S: Secretory pathway, i.e. the sequencecontains SP, a signal peptide; -: other location 


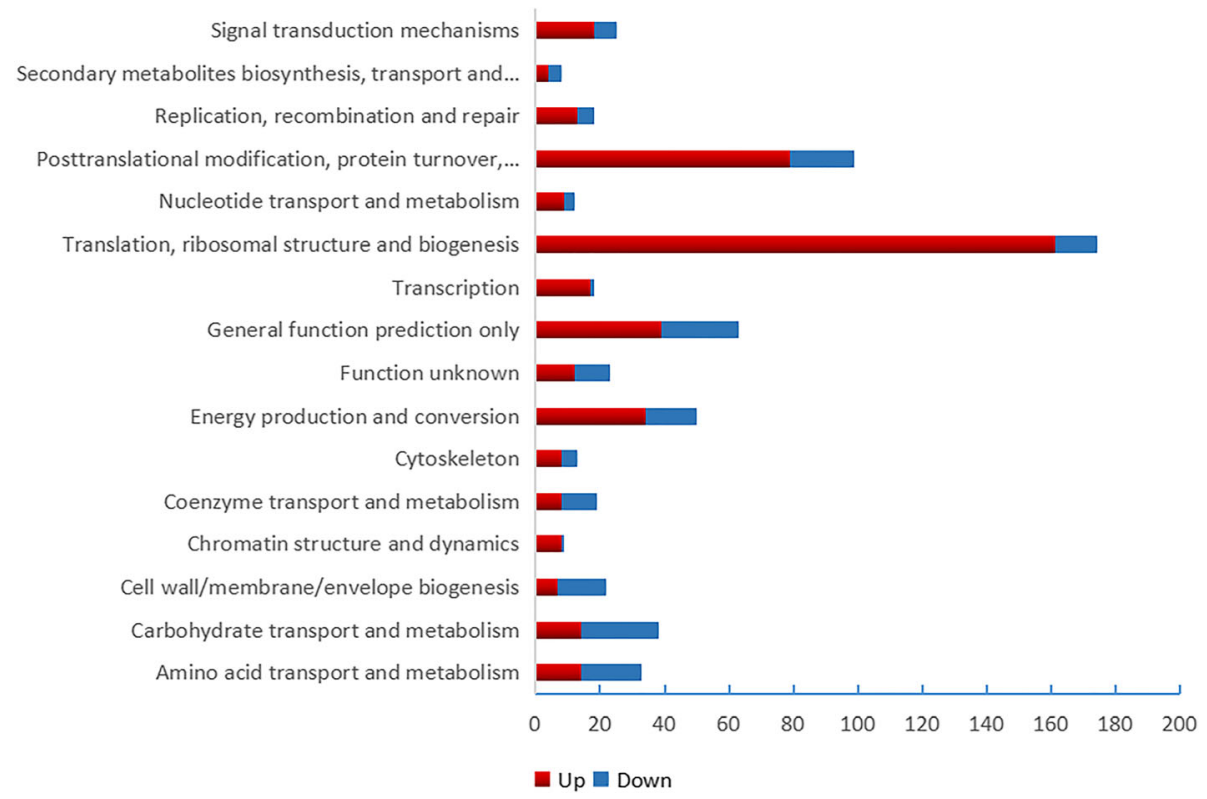

Fig. 3 COG function analysis DEPs of UpHT vs UpC. Colour in red indicated up-regulated proteins number, blue indicated down-regulated proteins number

which produces toxic compounds after reacting with the organic molecules. The glutathione-S-transferase (GST) enzymes play a significant role in detoxifying these toxins and help in their excretion or sequestration; the enzymes catalyse reduction of glutathione (GSH; a tripeptide Glu-Cys-Gly) by electrophilic and hydrophobic toxic molecules that are generated under stress to convert them to non-toxic and soluble conjugates [35]. The expression of glutathione S-transferase (Unigene15839_All,1.45-fold), MnSOD (CL6691_Contig2_All, 1.42-fold), ascorbate peroxidase (CL1454_Contig2_All,1.28-fold) and polyubiquitin (Unigene6725_All, 1.54-fold) were up-regulated. In addition, the expression of catalase-peroxidase (CL5101 Contig1_All, 0.66-fold), glutathione reductase (CL514 Contig1_All, 0.77-fold), and thioredoxin-dependent peroxidase (Unigene9950_All, 0.77-fold) were downregulated.

In addition, the $26 \mathrm{~S}$ proteasome regulatory subunit is a component of the hexameric ring of AAA-ATPases that forms the base of the $19 \mathrm{~S}$ regulatory particle (RP). This subunit plays specific roles in plant proteasomes by helping to promote the assembly of the RP with the $20 \mathrm{~S}$ core protease $(\mathrm{CP})$ and gate the $\mathrm{CP}$ to prevent indiscriminate degradation of cytosolic and nuclear proteins. In plants, this subunit plays an important role in diverse processes that include shoot and root apical meristem maintenance, cell size regulation, and stress responses [36]. In the present study, the $26 \mathrm{~S}$ proteasome regulatory subunit (CL1786_Contig3_All, CL2484_Contig2_All, 1.3 or 1.32-fold) was induced under high-temperature stress. The result is consistent with the observation that the expression of the $26 \mathrm{~S}$ proteasome subunit RPN10 is upregulated by salt stress in Dunaliella viridis [37]. The result indicated that the $26 \mathrm{~S}$ proteasome regulatory subunit played a key role in stress tolerance. Programmed cell death $(\mathrm{PCD})$ is an important biological phenomenon. Algae can activate PCD when exposed to external pathogens and other stresses. Metacaspases often play an important role in plant PCD, and a large number of experiments have shown that metacaspases are involved in plant PCD. In PCD, type II metacaspase was found to be transported from the cytoplasm to the nucleus, which promoted chromosome degradation. In the present study, mov34-domain-containing protein (Unigene14048_All, 1.33-fold) and metacaspase type II (CL6485_Contig1_All, 1.25 -fold) were up-regulated. The results indicated that the high-temperature stimulus accelerated the accumulation of ROS, inhibited the growth of $U$. prolifera, induced the expression of partial stress related proteins, and alleviated the stimulation. This process is consistent with the results that are reported for the brown alga Ectocarpus siliculosus [38].

\section{Expression analysis of DEPs that are involved in the oxidative phosphorylation pathway}

The oxidative phosphorylation (OXPHOS) process comprises an electron-transfer chain (ETC) that is driven by substrate oxidation and is coupled to the synthesis of ATP through an electrochemical transmembrane gradient. Oxidative phosphorylation is a vital part of metabolism, it also produces reactive oxygen species such as superoxide and hydrogen peroxide, which leads to propagation of free radicals, damages cells and contributes to disease, and possibly ageing. The previous study 
Table 3 KEGG pathway of DEPs(top15)(UpHT vs UpC)

\begin{tabular}{|c|c|c|c|c|}
\hline Pathway & $\begin{array}{l}\text { DEPs with pathway } \\
\text { annotation }\end{array}$ & $\begin{array}{l}\text { All Proteins with pathway } \\
\text { annotation (3046) }\end{array}$ & P value & Pathway ID \\
\hline \multicolumn{5}{|l|}{ Up-regulated } \\
\hline Ribosome & 134 & 179 & $2.73 E-66$ & ko03010 \\
\hline Oxidative phosphorylation & 32 & 84 & $1.18 \mathrm{E}-05$ & ko00190 \\
\hline Proteasome & 14 & 39 & 0.006672349 & ko03050 \\
\hline Glutathione metabolism & 15 & 46 & 0.01352303 & ko00480 \\
\hline SNARE interactions in vesicular transport & 5 & 10 & 0.0225611 & ko04130 \\
\hline Linoleic acid metabolism & 3 & 5 & 0.04546205 & ko00591 \\
\hline Protein processing in endoplasmic reticulum & 23 & 90 & 0.05135553 & ko04141 \\
\hline Phagosome & 13 & 45 & 0.05413349 & ko04145 \\
\hline Spliceosome & 25 & 101 & 0.06097134 & ko03040 \\
\hline Non-homologous end-joining & 2 & 3 & 0.08799354 & ko03450 \\
\hline RNA transport & 22 & 95 & 0.1338129 & ko03013 \\
\hline Porphyrin and chlorophyll metabolism & 12 & 47 & 0.1356993 & ko00860 \\
\hline Taurine and hypotaurine metabolism & 1 & 1 & 0.1828628 & ko00430 \\
\hline Vancomycin resistance & 1 & 1 & 0.1828628 & ko01502 \\
\hline Plant-pathogen interaction & 11 & 48 & 0.2512615 & ko04626 \\
\hline \multicolumn{5}{|l|}{ Down-regulated } \\
\hline Photosynthesis & 15 & 76 & 0.00047354 & ko00195 \\
\hline Carbon fixation in photosynthetic organisms & 15 & 83 & 0.001247104 & ko00710 \\
\hline Photosynthesis - antenna proteins & 8 & 31 & 0.001746649 & ko00196 \\
\hline Metabolic pathways & 91 & 952 & 0.005208609 & ko01100 \\
\hline Lysine biosynthesis & 3 & 11 & 0.04605148 & ko00300 \\
\hline Betalain biosynthesis & 1 & 1 & 0.07649376 & ko00965 \\
\hline Carotenoid biosynthesis & 4 & 23 & 0.09360185 & ko00906 \\
\hline Selenocompound metabolism & 3 & 17 & 0.1357731 & ko00450 \\
\hline Monobactam biosynthesis & 2 & 9 & 0.146953 & ko00261 \\
\hline Degradation of aromatic compounds & 1 & 2 & 0.1471594 & ko01220 \\
\hline Tyrosine metabolism & 3 & 18 & 0.1542241 & ko00350 \\
\hline Tryptophan metabolism & 3 & 19 & 0.1734048 & ko00380 \\
\hline One carbon pool by folate & 3 & 19 & 0.1734048 & ko00670 \\
\hline Insulin resistance & 4 & 29 & 0.1770334 & ko04931 \\
\hline
\end{tabular}

showed that low temperature, UVB radiation and phoxim-induced increase in oxidative phosphorylation gene [39-41]. In the present study, 34DEPs were mapped to the oxidative phosphorylation pathway (Figs. 5 and 6). The expression of 32 DEPs were found to be increased, and 2 DEPs were down-regulated. NADH:ubiquinone oxidoreductase (CL6587_Contig2_All), succinate dehydrogenase (CL2133_Contig2_All), cytochrome C oxidase subunit (CL1977_Contig2_All), and ATP synthesis subunit (gi|807046094|gb|AKC35152_1) were all significantly up-regulated by 1.36 fold, 1.59 fold, 1.23 fold, and 1.83 fold, respectively. The results are consistent with previous results. These results indicated that high-temperature induction enhanced the activity of the
ETC complex in the mitochondria. The imbalance between the activity changes of ETC may lead to the accumulation of reactive oxygen species.

\section{Expression analysis of DEPs involved in phytohormone and signal transduction pathways}

Studies have investigated the expression of the hormonerelated proteins that were changed under abiotic stress conditions [42]. In the present study, expression pattern analysis showed that the high-temperature stimulus changed the expression of key enzymes involved in phytohormone signal transduction and hormone biosynthesis. Approximately 12 DEPs were identified in phytohormone signal transduction (Fig. 7). In carotenoid biosynthesis, 

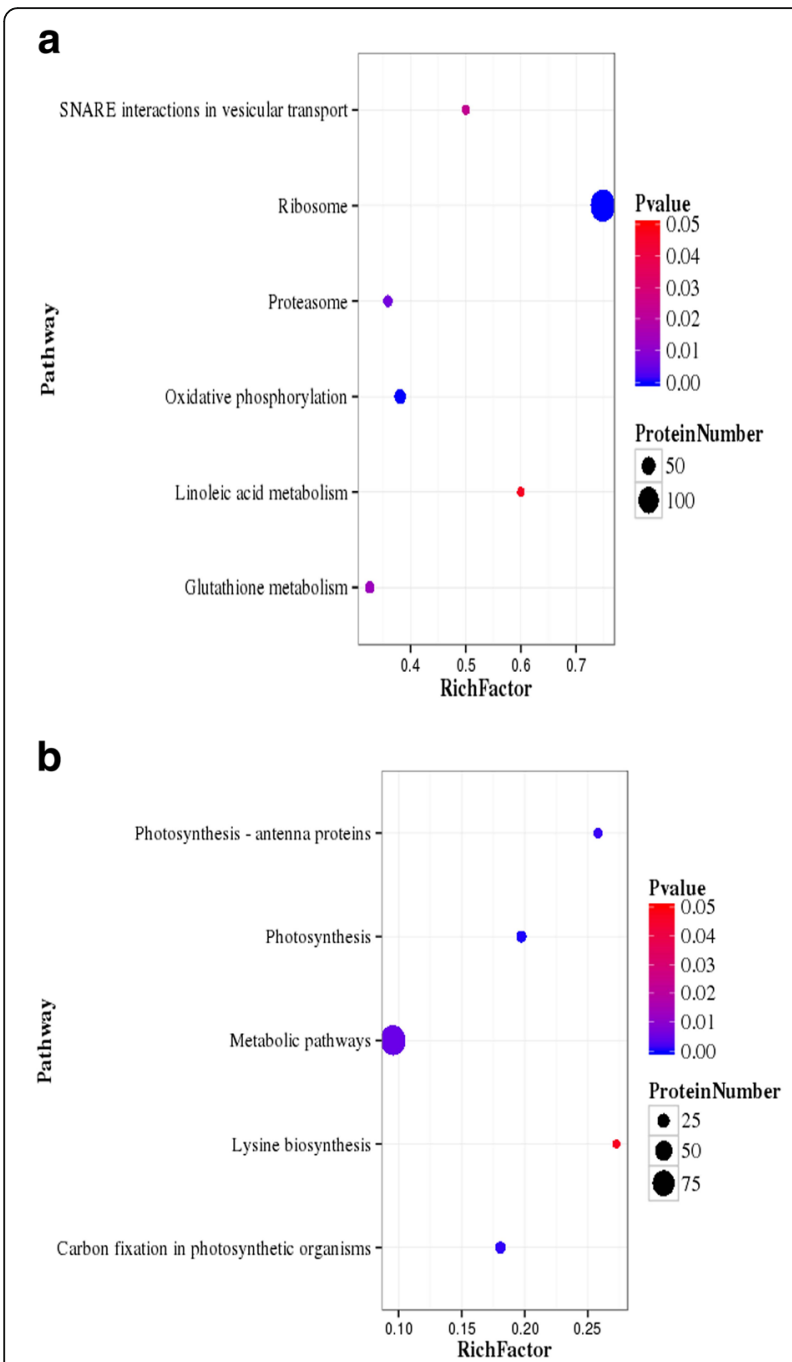

Fig. 4 Pathway enrichment statistical scatter plot of DEPs. a UpHT vs UpC up-regulated proteins $\mathbf{b} U \mathrm{pHT}$ vs UpC down-regulated proteins. The vertical axis represents the name of the pathway; the horizontal axis represents the pathway corresponding Rich factor. The colour indicated $p$ value. Rich factor refers to the ratio of the number of differentially expressed genes in the pathway and the number of all annotated genes in the pathway. Higher Rich factors indicate greater degrees of enrichment. $Q$ values are often completed after multiple hypothesis testing with corrected $P$ value values ranging from 0 to 0.05 . The closer they are to zero, the more significant the enrichment

cytochrome P450 (Unigene18531_All) was significantly up-regulated by 1.23 fold. The expression of violaxanthin de-epoxidase-related protein (CL1646_Contig1_Alland CL1850_Contig11_All), prolycopene isomerase (Unigene205_All) and zeaxanthin epoxidase (CL4385_Contig1_All) were down-regulated by 0.83 fold, 0.74 fold, 0.81 fold, and 0.82 fold compared to the control. In brassinosteroid (BR) biosynthesis, molecular and biochemical studies revealed that two different cytochrome P450s, CYP90B2 and CYP724B1/D11, are the rate-limiting step of brassinosteroid biosynthesis. Three significant DEPs that were mapped to the $B R$ synthesis pathway the expression of cytochrome P450s 90B/724B (gi|158273526|gb|EDO99315_1|, 0.69-fold) were decreased. The expression of 90C1D1 (CL3922_Contig1_All, 1.29-fold) and CYP735A (Unigene18531_All, 1.23-fold) were up-regulated. CYP735A encodes cytokinin hydroxylases that catalyse iP-nucleotide converts to trans-Zeatin. The signalling transduction of the cytokinin pathway and abscisic acid (ABA) pathway played an important role in the growth and development of plants and the regulation of stress resistance. AHP (Histidine-containing phosphotransfer protein) mainly functions as two-component phosphorelay mediators between cytokinin sensor histidine kinases and response regulators (B-type ARRs) and plays an important role in promoting cytokinin signal transduction via the multistep His-to-Asp phosphorelay. Biochemical and molecular genetic studies in Arabidopsis have identified protein phosphatase 2C (PP2C) enzymes as negative regulators in plant signal transduction processes [43]. In the absence of ABA, PP2C mainly inhibited the active state of SnRK2s protein. The inhibition of the active state of SnRK2s protein result in a decrease in downstream transcriptional regulation. ABA relieved the inhibition effect of PP2C on SnRK2s, activated the SnRK2s protein, and initiated downstream signal transduction. In the present study, the expression of the AHP protein (CL7329_Contig1_All, 1.25-fold) and SnRK2s (CL4735_ Contig1_All, 1.27-fold) protein was up-regulated. However, the expression of PP2C (CL5191_Contig2_All, 0.8-fold) was down-regulated, which indicated that the signal transduction pathway of cytokinin and ABA was induced at a high temperature. The result is consistent with previous rzesearch [42, 43]. Plant growth is co-ordinately regulated by environmental and hormonal signals. Brassinosteroid (BR), cytokinin and $\mathrm{ABA}$ played essential roles in growth regulation via light and temperature; however, the interactions between hormone signal transduction and environmental signals remain poorly understood at the molecular level. In summary, the results indicated that phytohormone signal regulation played a key role in the high-temperature stimulus and enhanced cytokinin and ABA signal transduction pathway. However, the regulation mechanism should be further considered.

\section{Expression analysis of DEPs involved in photosynthesis- related proteins}

Photosynthesis is very sensitive to high-temperature stress. Changes in environmental temperature are primarily reflected by photosynthesis, which triggers a response aimed at attaining the best possible performance under the new conditions. Several studies have shown that high-temperature stress can significantly inhibit photosynthesis $[44,45]$. In green algae, light-harvesting chlorophyll protein complexes are the 


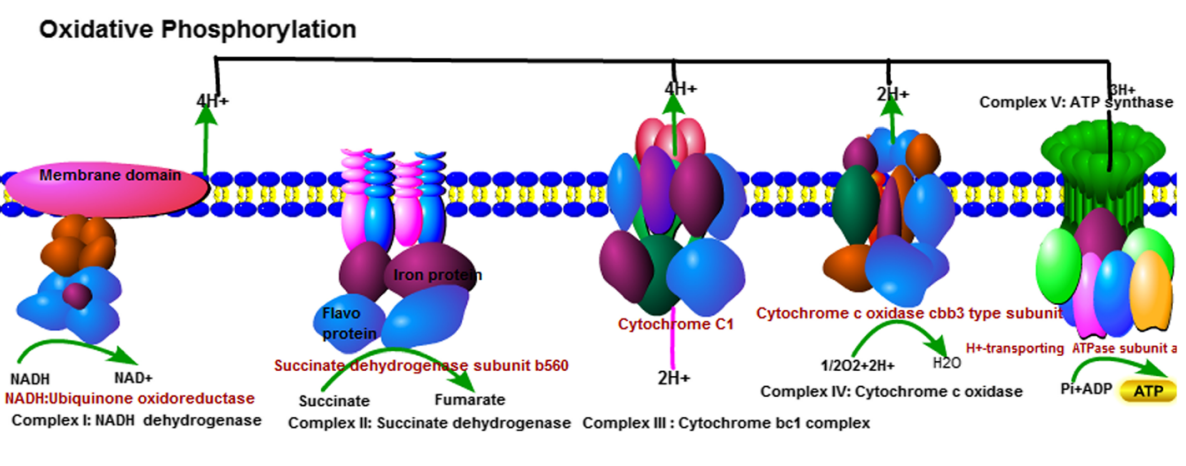

Fig. 5 The oxidative phosphorylation pathway

major light-harvesting complexes. These complexes include photosystem II (light-harvesting complex II and photosynthetic reaction centre) and photosystem I (LHCI and photosynthetic reaction centre). The light-harvesting complex is a complex of subunit proteins that may be part of a larger supercomplex of the photosystem, and the function is to collect more of the incoming light. In the previous study, LHCI of photosystem I Chlamydomonas reinhardtii is composed of nine genes. Three product complexes (Lhca1, 3 and 7) showed emission peaks in the

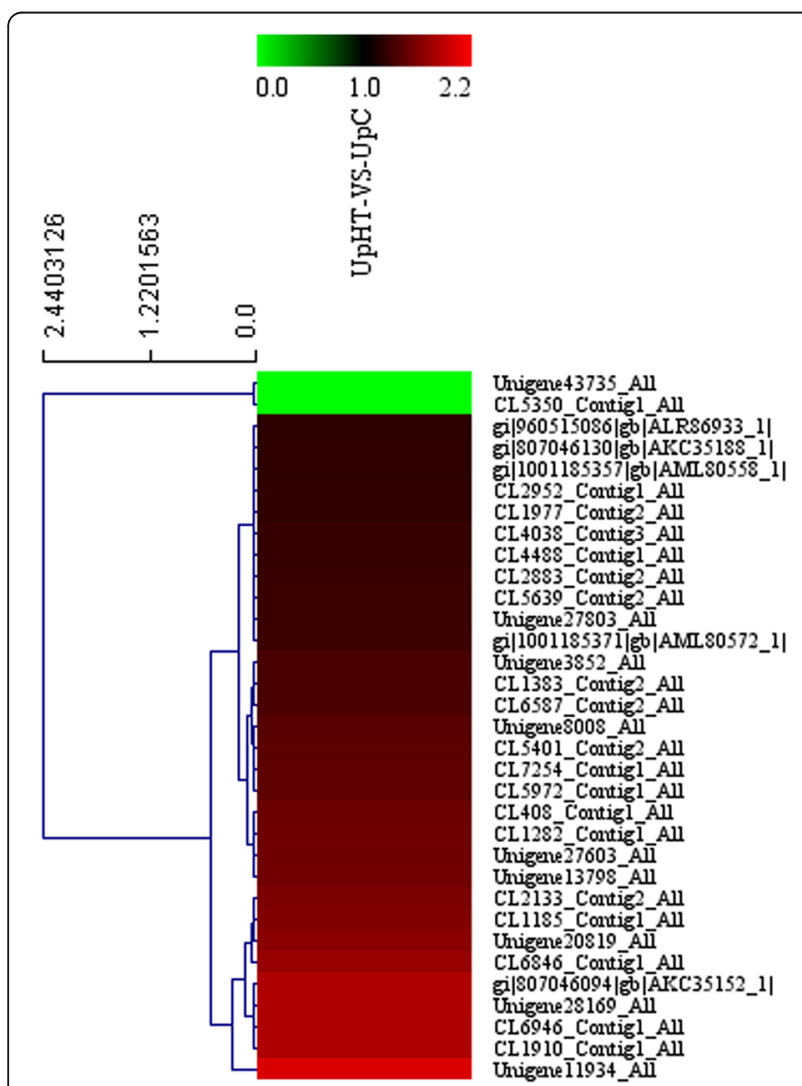

Fig. 6 The cluster of oxidative phosphorylation pathway(UpHT vs UpC). Red indicated the expression of protein up-regulated, green indicated the expression of protein down-regulated range of $680-690 \mathrm{~nm}$, thus accounting for the blue contribution observed in the native preparations. Three other complexes (Lhca5, 6 and 8) exhibit emission in the 695$700 \mathrm{~nm}$ range. This emission component is not clearly resolved in the fluorescence spectra of the native LHCI preparations, possibly due to energy transfer to the red forms of a neighbouring complex or due to a change in the absorption properties of these complexes, because of protein-protein interactions, when embedded in the PSILHCI supercomplex, as previously proposed [46]. Three more complexes, namely, Lhca2, 4 and 9, exhibited emission above $707 \mathrm{~nm}$ with the red-most form $(715 \mathrm{~nm})$ associated with Lhca2, which indicates that Lhca2 is responsible for the red-most emission in vivo. Up-regulation of Lhca4 and Lhca9 was observed under iron-deficiency conditions, and it correlates with a shift to the blue end of the emission spectrum (from 710 to $704 \mathrm{~nm}$ ) and with a relative increase in intensity of the emission above $700 \mathrm{~nm}$, compared to that for the emission below $700 \mathrm{~nm}$ [47]. This indicates an increase in the content of red forms that emit approximately $705 \mathrm{~nm}$ in the antenna, in agreement with the fluorescence characteristics of Lhca4 and Lhca9. Under the same conditions, down-regulation of Lhca5 was also detected, leadin to the suggestion that Lhca4 can substitute for Lhca5, which possibly provides a better sink for energy dissipation. The finding that these antennas contain the lowest energy forms supports the hypothesis. A similar effect was recently observed in higher plants, where Lhca5 could substitute for Lhca4 [48, 49]. In the present study, the LHCI subunits lhca1 (CL4203_Contig1_All, 0.72-fold) and lhca3 (CL654_Contig2_All, 0.8-fold) were down-regulated, and lhca2 (LhcSR, gi|315319013|gb|ADU04518_1|, 1.3 -fold) was up-regulated. It may be possible that the decrease in the emission of blue light results in energy transfer to the red forms. Lhca2 is responsible for the red-most emission. The up-regulation of lhca2 indicated that the lowest energy forms were native organized. Lhca1 and lhca3 were unstable and underwent decomposition under high temperature. The 


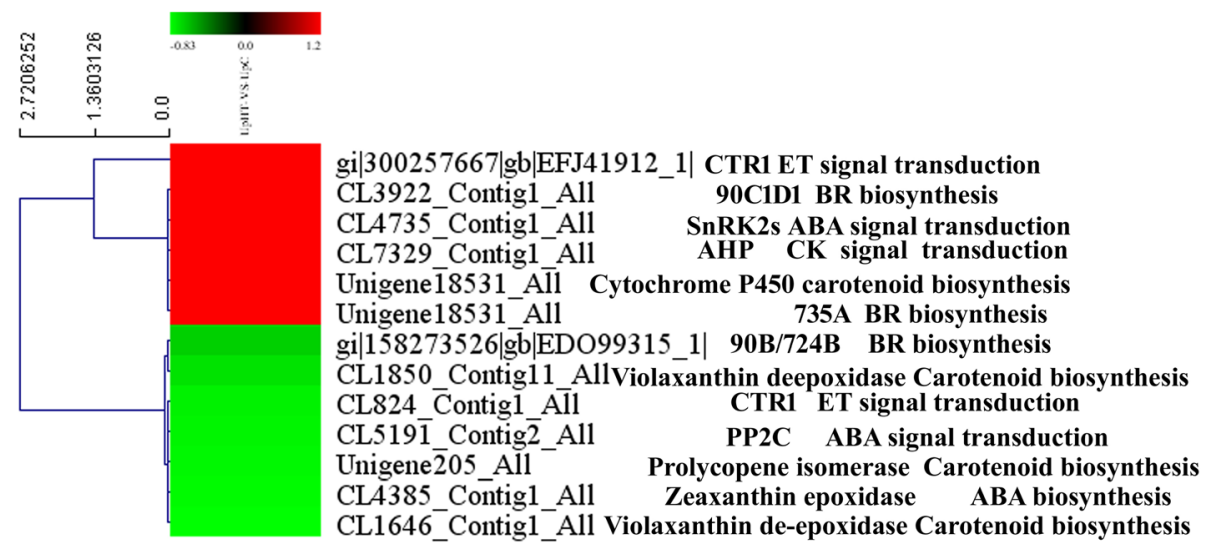

Fig. 7 The cluster of plant hormone biosynthesis and signal transduction related proteins(UpHT vs UpC). Red indicated the expression of protein up-regulated, green indicated the expression of protein down-regulated

results were consistent with that obtained by Naumann et al. [47]. In addition, the two most abundant light-harvesting complex II are Lhcb1 and Lhcb2, which make up light-harvesting complex (LHC) II trimers. They are also involved in facilitating state transitions, a process during which energy balance between photosystem (PS) II and I is achieved. In the present study, the LHCII subunits lhcb1 and lhcb2 were also down-regulated. Down-regulation of LHCII would significantly influence light absorbance and energy transfer in $U$. prolifera upon a hightemperature stimulus (Fig. 8). The results were consistent with the observation that salt stress induces a decrease in excitation energy transfer from phycobilisomes to photosystem II but an increase to photosystem I in the cyanobacterium Spirulina platensis [50]. Eighteen DEPs were mapped to the photosynthesis pathway, and the expression of 14 DEPs were down-regulated. Ferredoxin-NADP ${ }^{+}$reductase (FNR) is one of the important enzymes involving in many biochemical and physiological metabolism processes, such as electron transfer, carbon dioxide fixation, nitrogen assimilation and antioxidation. Ferredoxin-NAD$\mathrm{P}^{+}$reductase (Unigene9738_All, 0.82-fold and CL7087_Contig1_All, 0.81-fold) and photosystem I and photosystem II subunit proteins were identified and found to be decreased.

However, the expression of the chloroplast ATP synthase subunit that was encoded by 5 unigenes (gi|807,046,094|gb|AKC35152_1|, 1.82-fold) was up-regulated.

This result indicated that the high temperature inhibited photosynthesis of $U$. prolifera and induced stress-related LhcSR protein expression, which was consistent with the expression of LhcSR in $U$. linza in a study that was conducted by Dong et al. [51]. The electrons from PSI and PSII might transfer to oxygen, and thereby lead to substantial generation of reactive oxygen species. Enrichment of the oxidative phosphorylation pathway under cold treatment suggested that high-temperature induced ROS production might occur. However, this hypothesis still lacks experimental evidence.

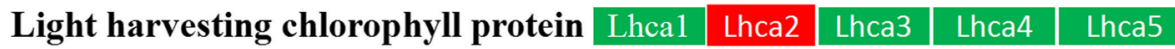

\begin{tabular}{|c|c|c|c|c|c|}
\hline \multirow{2}{*}{ Photosystem II } & PsbA & PsbD & PsbC & PsbO & PsbP \\
\hline & PsbQ & PsbS & Psb27 & & \\
\hline Photosystem I & PsaC & PsaJ & PsaL & & \\
\hline & $\mathrm{PC}$ & FNR & & & \\
\hline Photosynthetic electron transport & PetE & PetH & & & \\
\hline F-type ATPase & $\boldsymbol{\alpha}$ & Y & $\varepsilon$ & $\delta$ & b \\
\hline
\end{tabular}

Fig. 8 The expression of photosynthesis-related proteins (UpHT vs UpC). Red indicated the expression of protein up-regulated, green indicated the expression of protein down-regulated 


\section{Expression analysis of DEPs involved in the carbon-fixation pathway}

According to $\mathrm{CO}_{2}$ assimilation mechanisms, plants can be classified into three types: $\mathrm{C}_{3}, \mathrm{C}_{4}$, and CAM. $\mathrm{C}_{4}$ plants have acquired a series of high-performance photosynthetic genes during evolution, which confer more efficient photosynthesis than that in $\mathrm{C}_{3}$ plants under adverse conditions such as high light intensity, temperature, and low $\mathrm{CO}_{2}$ concentration. In the present study, 19 DEPs were mapped to carbon fixation in photosynthetic organisms (Fig. 9). The expression of 14 DEPs was down-regulated, and that of 5 DEPs was up-regulated. In $\mathrm{C}_{4}$-dicarboxylic acid cycle, the key regulated enzymes aspartate aminotransferase (Unigene42677_All, 0.71-fold), pyruvate orthophosphate dikinase (Unigene19826_All, 0.73-fold), and NADP malic enzyme (CL5095_Contig3_All, 0.81-fold) were down-regulated. At the same time, pyruvate orthophosphate dikinase (Unigene19826_All, 0.73-fold) and NADP malic enzyme were also found to be down-regulated in CAM. Moreover, the expressions of plastid transketolase (Unigene20747_All, 0.43-fold), fructose-1,6-bisphosphatase (CL438_Contig1_All, 0.81-fold), glyceraldehyde-3-phosphate dehydrogenase (CL7324_Contig1_All, 0.72-fold) and phosphoglycerate kinase (CL1803_Contig1_All, 0.81-fold) were down-regulated. Ribulose-1,5-bisphosphatecarboxylase/oxygenase large subunit was found to be encoded by 7 DEPs, of which, 4. DEPs were up-regulated, and 3 DEPs were down-regulated. The result demonstrated that the expression of the key enzyme in the carbon-fixation pathway was down-regulated.

The activation of rubisco in vivo requires the presence of the regulatory protein rubisco activase. This enzyme facilitates the release of sugar phosphate inhibitors from rubisco catalytic sites thereby influencing carbamylation. Rubisco activase was used to explore the role of the enzyme in $\mathrm{C}_{4}$ photosynthesis at a high temperature. It is concluded that during short-term treatment at $40{ }^{\circ} \mathrm{C}$, rubisco activase content is not the only factor that modulates rubisco carbamylation during $\mathrm{C}_{4}$ photosynthesis [52]. In the present study, rubisco activase (CL649_Contig6_All, 1.32-fold) was up-regulated; however, it is not the only factor. At the same time, soluble carbonic anhydrase precursor encoded by CL7120_Contig2_All was down-regulated, and CL6328_Contig1_All was up-regulated. In other words, to protect the energy balance, $U$. prolifera decreased photosynthesis and carbon fixation of metabolic activity under hightemperature conditions. However, increase in the expression of carbonic anhydrase precursor and rubisco activase play an important role in the acceleration to photosynthesis balance adjustment.

\section{Expression analysis of DEPs involved in the glutathione metabolism pathway}

A total of 18 DEPs were mapped to the glutathione metabolism pathway. The expression of key enzymes in glutathione metabolism pathway were observed to significantly change. Glutathione S-transferase (GST) catalyses the nucleophilic fusion of reduced glutathione (GSH; a tripeptide Glu-Cys-Gly) with electrophilic and hydrophobic toxic molecules, which were generated under stress, to convert them to non-toxic and soluble conjugates. GSTs play an important role in various metabolic pathways, and they are involved in detoxification of oxidative lipid peroxide metabolites, hormone metabolism, stress resistance, protection of cell oxidative stress injury, cell signal transduction and disease resistance [53-55]. Ascorbate peroxidase (APXs) catalyses the $\mathrm{H}_{2} \mathrm{O}_{2}$-dependent oxidation of ascorbate in plants, algae and certain cyanobacteria. Glutathione reductase (GR)

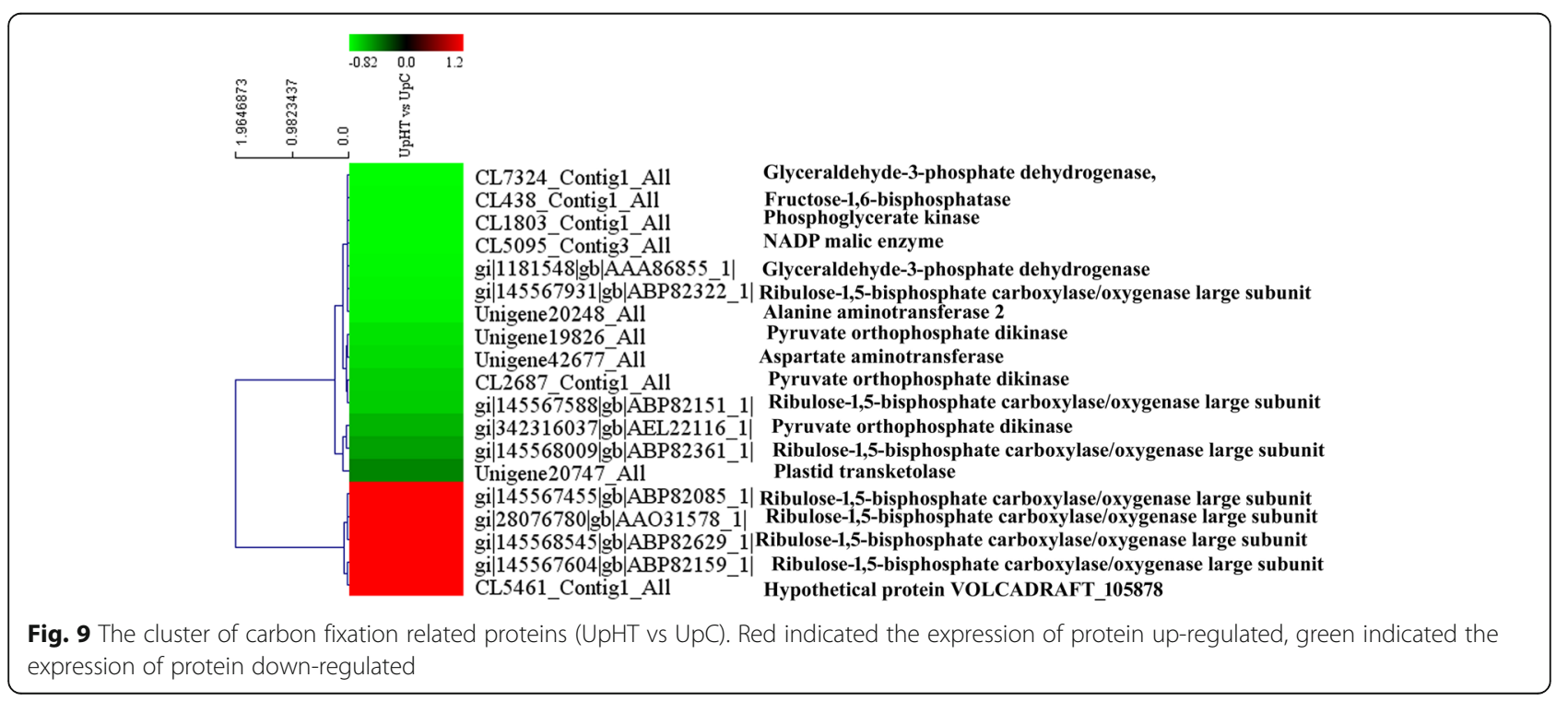


catalyses the reduction of glutathione disulphide (GSSG) to the sulphydryl form glutathione (GSH), which is a critical molecule in resisting oxidative stress and maintaining the reducing environment inside the cell [56-58]. In the present study, APX (CL1454_Contig2_All, 1.28-fold), glutathione S-transferase (CL2243_Contig1_All, 1.53-fold), glucose-6-phosphate 1-dehydrogenase 2(CL2243_Contig1_All, 1.53-fold) and 6-phosphogluconate dehydrogenase (gi|158274793|gb|EDP00573_1|, 1.32-fold), decarboxylating(gi|158274793|gb|EDP00573_1|, 1.32-fold) were found to significantly increase. However, the expression of GR(CL514_Contig1_All, 0.77-fold) was down-regulated (Fig. 10). It is indicated that high temperature stimulus accumulated more ROS, induced the expression of resistance-related protein glutathione S-transferase and APX.

\section{Expression analysis of $\mathrm{Ca}^{2+}$-binding protein}

$\mathrm{Ca}^{2+}$-dependent signalling processes are beneficial to plant perception, and they respond to diverse environmental stressors, such as osmotic stress and high temperature [59-61]. In our study, the expression of Hsp90 (Unigene40313_All, 1.78-fold), CDPK (Unigene26962_All, 1.35-fold) and EDS1 (gi|158,282,407|gb|EDP08159_1|, 1.4-fold) was up-regulated in $U$. prolifera under the high-temperature stimulus. In addition, CDPK can activate $\mathrm{H}_{2} \mathrm{O}_{2}$ accumulation through regulating NADPH oxidase. The results indicated that the high-temperature stimulus accelerated ROS signalling, CDPK and EDS1 expression. In conclusion, the $\mathrm{Ca}^{2+}$-signal also played an important role in high-temperature stress in $U$. prolifera.

\section{Expression analysis of DEPs involved in other types of} metabolisms

Linoleic acid (LA), a carboxylic acid, belongs to one of the two families of essential fatty acids. It is converted by various lipoxygenases and cyclooxygenases and the cytochrome P450 enzyme. All of these LA products exhibit bioactivity, and they are implicated in human physiology and pathology as indicated in the cited linkages. In the present study, 4 DEPs were mapped to linoleic acid metabolism. The expression of the alpha-beta hydrolase superfamily (gi|158279131|gb|EDP04893_1|, 2.05-fold), cytochrome P450 (Unigene18531_All, 1.23-fold), lipoxygenase (Unigene17329_All, 3.21-fold), and 12-oxophytodienoic acid reductase (CL2323_Contig1_All, 1.47-fold) was up-regulated. In addition, glycan, taurine, and hypotaurine played a key role in plant response to stress. The expression of glycan-biosynthesis-related proteins changed significantly under high-temperature stimulus. The expression of dolichyl-phosphate beta-glucosyl transferase (ALG ${ }_{5}$, Unigene5913_All, 1.29-fold) was up-regulated. However, alpha-1,2-mannosyltransferase (ALG9, CL1555_Contig1_All, 0.78-fold) was down-regulated. Glycosyl transferase is encoded by two DEPs; one is down-regulated, and the other is up-regulated. Moreover, it has been suggested that taurine, hypotaurine and their metabolic precursors (cysteic acid, cysteamine and cysteine sulphinic acid) might act as antioxidants through scavenging $\cdot \mathrm{OH}, \mathrm{HOCl}$ and $\mathrm{H}_{2} \mathrm{O}_{2}$. It is concluded that cysteamine and hypotaurine are far more likely to act as antioxidants in vivo than taurine, provided that they are present in a sufficient concentration at sites of oxidant generation [62]. In our study, the expression of cystathionine beta-synthase (Unigene42486_All, 1.47-fold) was observed to be up-regulated. This result indicates that the

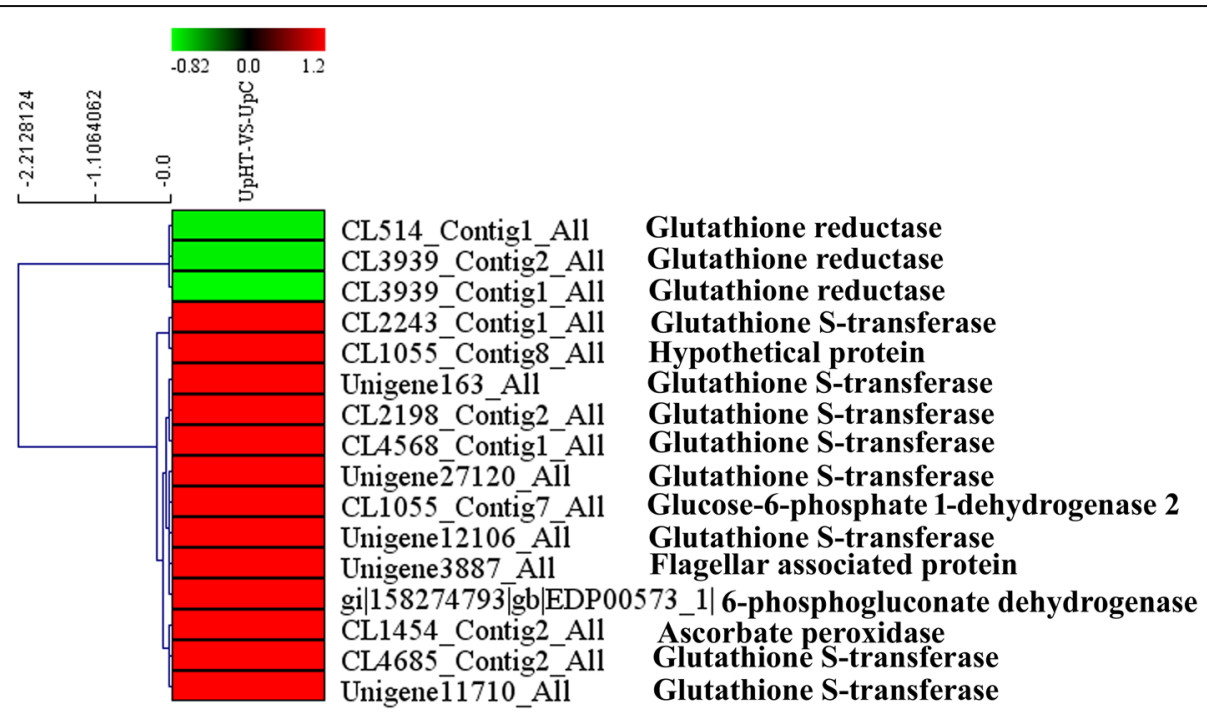

Fig. 10 The cluster of glutathione metabolism related proteins (UpHT vs UpC). Red indicated the expression of protein up-regulated, green indicated the expression of protein down-regulated 
high-temperature stimulus induced cysteamine biosynthesis and increased the antioxidant levels.

\section{qPCR to verify the reliability of the proteome}

The expression of 8 unigenes were investigated via qPCR. The results were consistent with the available proteome data (Additional file 1: Table S1). Quantitative real time polymerase chain reaction results indicated that the unigenes that encode heat shock protein 90, APX, MnSOD, glutathione reductase, glutathione S-transferase, RbcL, heat shock protein 70 and ATP synthase were all significantly up-regulated by $3.152,2.502,2.106,0.420,2.320$, $2.522,2.242$ and 3.252 -fold, respectively.

\section{Validation of differentially expressed proteins identified by western blot}

Two proteins, Hsp70 and RbcL identified DEPs with marked differences in expression determined by iTRAQ based quantitative analysis were selected to be verified by western blot analysis (Fig. 11). Hsp70 and RbcL protein was significantly up-regulated in UpHT groups as compared with control group $(p<0.05)$. The results which were found by western blot is consistent with the findings in iTRAQ analysis. It is well established that Hsp70 and RbcL could play a role in protecting the protein structure and photosynthesis early in the stress response.

\section{PPI network}

In plants, proteins do not function in isolation within cells but as part of a network. In this study, a PPI network was generated to highlight the interactions and relationships between different proteins. To obtain interaction between proteins, we constructed a PPI network that was based on data downloaded from the STRING

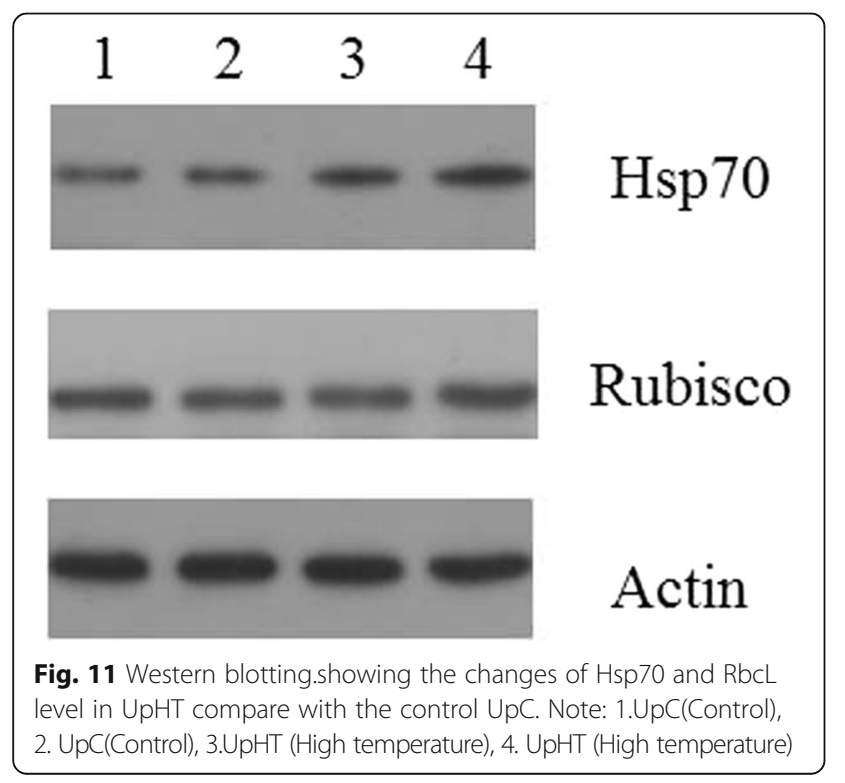

database. For the UpHT vs UpC protein, we chose 102 proteins that were involved in response to stimulus, photosynthesis, carbon fixation in photosynthetic organisms, oxidative phosphorylation, glutathione metabolism, plant signal transduction and plant-pathogen interaction to construct the PPI. Based on the differential expression patterns at the protein levels in UpHT compared to UpC (Fig. 12). In the present study, there are 37 unigenes nodes and 194 interactions in the PPI network. The PPI network indicated that heat shock protein 90C (CL2081_Contig1_All, 1.49-fold), heat shock protein 90C (gi|158270891|gb|EDO96722_1|, 1.44-fold), heat shock protein 70 (gi|219764948|emb|CAW63927_1|, 1.4-fold), ClpB chaperone, Hsp100 family (CL517_Contig2_All and Unigene15739_All, 1.64-fold and 1.46-fold) and heat shock protein 70B (gi|297592060|gb|ADI46845_1|, 1.82-fold) are involved in interactions with proteins from other functional groups and played important signal regulated. In addition, soluble inorganic pyrophosphatase 2 (CL408_Contig1_All, 1.51-fold), plastid transketolase (Unigene20747_All, 0.43-fold), F1F0 ATP synthase gamma subunit (Unigene20819_All, 1.69-fold), ribulose-1,5-bisphosphate carboxylase/oxygenase large subunit (gi|323,320,892|gb|ADX36432_1|, 0.8 -fold), serine/threonine-protein kinase CTR1 (gi|300257667|gb|EFJ41912_1|, 2.66-fold), component of cytosolic $80 \mathrm{~S}$ ribosome and $40 \mathrm{~S}$ small subunit (CL2825_Contig3_All, 1.3-fold), flagellar associated protein (CL4919_Contig2_All, 1.53) as the key regulation factor and construct PPI under high temperature.

\section{A pathway model of high temperature stress responses in U. prolifera}

Using our results and previous studies, we propose a putative synergistic response network for $U$. prolifera that responds to high-temperature stress. As shown in Fig. 13, the reaction of $U$. prolifera to high-temperature stress rapidly accumulates ROS, which leads to a series of metabolic changes. First, high-temperature stress induces stress signals to generate differential accumulation of signal transduction components. High temperature causes the cell ROS concentration to rapidly increase, and high concentrations of ROS result in an increasing release of $\mathrm{Ca}^{2+}$ into the cytoplasm. When the concentration of $\mathrm{Ca}^{2+}$ reaches a particular threshold, CDPK is activated. As a result, plants are able to produce appropriate responses to the stress conditions. In an emergency response, HSP, as the key molecule, is significantly up-regulated to protect proteins from misfolding. To remove the accumulated ROS, the antioxidant system is activated, and proteins that regulate the redox balance, including MnSOD, GSTs, APX and other response factors, are up-regulated. These proteins can break down excessive intracellular ROS and restore the 

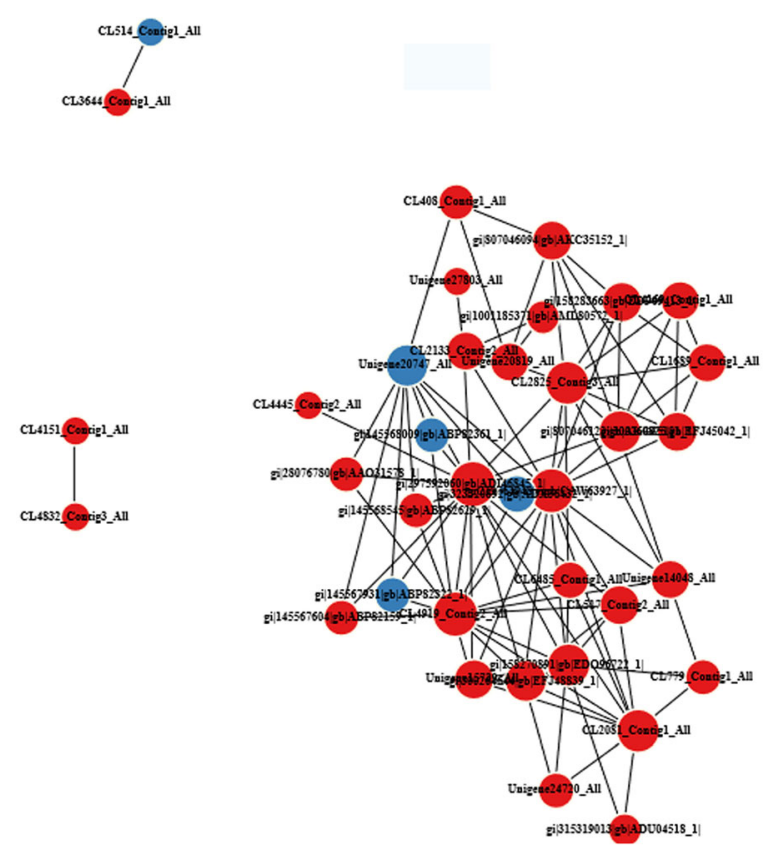

Fig. $12 \mathrm{PPI}$ network of U. prolifera response to high temperature stimuls. Red indicated the expression of protein up-regulated,blue indicated the expression of protein down-regulated. CL2081_Contig1_All: heat shock protein 90C; gi|158270891|gb|EDO96722_1|:heat shock protein 90C; gi|219764948|emb|CAW63927_1|:heat-shock inducible Hsp70;Unigene20819_All:F1F0 ATP synthase gamma subunit; CL517_Contig2_All:ClpB chaperone, Hsp100 family; gi|1001185371|gb|AML80572_1|:NADH-ubiquinone oxidoreductase 49 kDa subunit; CL4445_Contig2_All:prohibitin; gi|315319013|gb|ADU04518_1|:LhcSR; gi|300264644|gb|EFJ48839_1|:Stress-induced-phosphoprotein; CL408_Contig1_All:soluble inorganic pyrophosphatase 2; gi|300257667|gb|EFJ41912_1|:Serine/threonine-protein kinase CTR1;CL2825_Contig3_All:component of cytosolic 80S ribosome and 405 small subunit;Unigene15739_All:ClpB chaperone, Hsp100 family; CL4169_Contig1_All:nucleolar protein, component of C/D snoRNPs; Unigene20747_All:plastid transketolase; Unigene24720_All:DNA binding helix-turn helix protein; CL2133_Contig2_All:iron-sulfur subunit of mitochondrial succinate dehydrogenase;gi|297,592,060|gb|ADI46845_1|:heat shock protein 70B;CL4919_Contig2_All:flagellar associated protein;gi|300260825|gb|EFJ45042_1|:component of cytosolic 80S ribosome and 60S large subunit; CL7329_Contig1_All:histidine-aspartic acid phosphotransferase 1;CL4832_Contig3_All:ATP-dependent Clp protease regulatory subunit ClpC

normal intracellular redox environment. Proteins with important functions in energy metabolism are continuously upregulated to generate ATP, which allows plants to perform vital metabolic functions and to combat stress. Oxidative phosphorylation is the major approach to generate ATP, accompanied by the accumulation of ROS. The high-temperature stimulus induced upregulation of electron transport chain complex protein: NADH:ubiquinone oxidoreductase, succinate dehydrogenase, cytochrome C oxidase subunit, and ATP synthesis subunit in mitochondria. The imbalance between the activity changes of ETC may lead to accumulation of reactive oxygen species. The possible reason was that changes in the quinone pool redox state are responsible for the apparent inorganic phosphate activation of complex III. Complex III is responsible for higher ROS production during physiological working conditions relative to complex I [63]. At the same time, protein synthesis increased, and cytosolic $80 \mathrm{~S}$ ribosome and $40 \mathrm{~S}$ small subunit, nucleolar protein, and component of C/D snoRNPs were all up-regulated. However, the effect of high temperature on proteins involved in photosynthesis and carbon fixation are downregulated, and the result was consistent with the proteome response of Brachypodium distachyon to drought stress [64].

\section{Biological index and photosynthesis index}

Antioxidant enzymes played an important role in preventing the oxidation of biological molecules and protecting plant tissues from damage caused by ROS that was induced by multiple environmental stresses. SODs catalysed $\mathrm{O}^{2-}+2 \mathrm{H}^{+} \rightarrow \mathrm{O}_{2}+\mathrm{H}_{2} \mathrm{O}_{2}$. In addition, $\mathrm{APX}$ and CAT catalysed $\mathrm{H}_{2} \mathrm{O}_{2} \rightarrow \mathrm{O}_{2}+\mathrm{H}_{2} \mathrm{O}$. In the present study, the effects of high temperature were investigated on the activity of antioxidant enzymes, the chlorophyll content, and the chlorophyll fluorescence parameter of $U$. prolifera. The result indicated that under a high-temperature stimulus, the protein content decreased during the all process compared to the control group (Additional file 1: Figure S1). SOD activity was decreased under high temperature compared to the control group. However, the APX activity increased in the later period (Additional file 1: Figure S2, Fig. 4). The MDA content increased over the time and reached the highest value after $96 \mathrm{~h}$, which was significantly different from the 


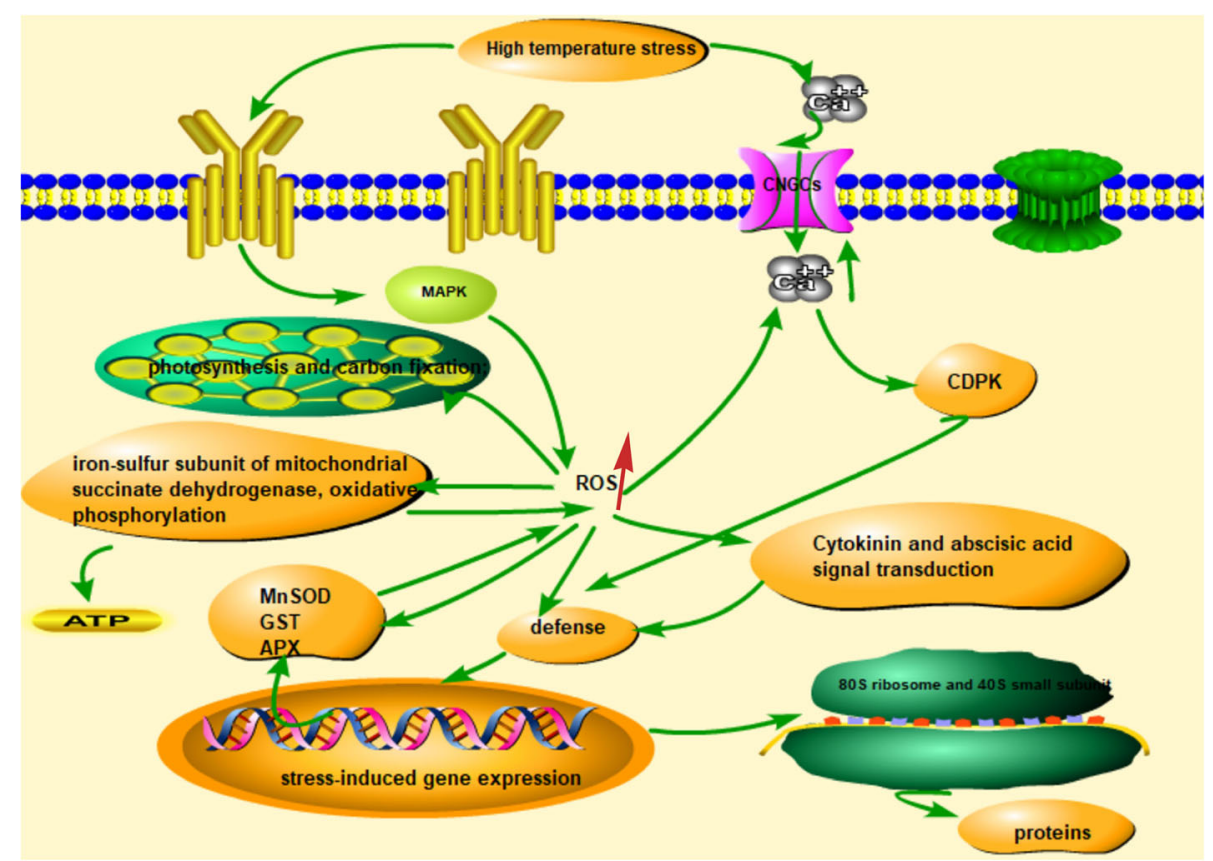

Fig. 13 A pathway model of high temperature stress responses in U. prolifera. MAPK: mitogen-activated protein kinase; CNGC:Cyclic-nucleotide gated channel;CDPK:Calcium-dependent protein kinases;ROS: reactive oxygen species;APX: ascorbate peroxidase;GST:glutathione S-transferase;MnSOD: manganese superoxide dismutase

control group (Additional file 1: Figure S3). Furthermore, the value of $\mathrm{Fv} / \mathrm{Fm}$ dropped to the lowest point after $96 \mathrm{~h}$ of culture (Additional file 1: Figure S5). Our results provide important information for the selection of heat-resistant $U$. prolifera.

\section{Conclusions}

A total of 1223 differentially expressed proteins were identified in response to heat stress. The up-regulated proteins were enriched in ribosome, oxidative phosphorylation, proteasome, glutathione metabolism, SNARE interactions in vesicular transport and linoleic acid metabolism pathway. The down-regulated proteins were in photosynthesis, carbon fixation, photosynthesis antenna proteins, and metabolic pathways. The up-regulated proteins were mainly glutathione S-transferases, heat shock proteins, manganese superoxide dismutase, ubiquitin-related protein, lhcSR, rubisco activase, serine/threonine protein kinase 2, adenylate kinase, $\mathrm{Ca}^{2+}$-dependent protein kinase (CDPK), disease resistance protein EDS1, mov34-domaincontaining protein, metacaspase type II, NDPK2a, 26S proteasome regulatory subunit, ubiquinone oxidoreductase, ATP synthase subunit and cytochrome $\mathrm{C}$ oxidase subunit AHP, SnRK2s, and cytochrome P450. The down-regulated proteins were photosynthesis-related proteins, glutathione reductase, thioredoxin, thioredoxin peroxidase and carbon-fixation-related proteins. Overall, stress response, oxidative phosphorylation, cytokinin signal transduction pathway, abscisic acid signal transduction pathway, and $\mathrm{Ca}^{2+}$ signal transduction played key roles in high-temperature regulation. Moreover, high temperature might lead to photosynthesis and inhibition of carbon fixation in $U$. prolifera. These novel proteins provide a good starting point for further research into their functions using genetic or other approaches. These findings significantly improve the understanding of the molecular mechanisms that are involved in the tolerance of algae to high-temperature stress.

\section{Additional files}

Additional file 1: Figure S1. Effects of high temperature on protein content of U. prolifera. Figure S2. Effects of high temperature on superoxide dismutase activity of U. prolifera. Figure S3. Effects of high temperature on MDA contents of U. prolifera. Figure S4. Effects of high temperature on APX activity of U. prolifera. Figure S5. Effects of high temperature on the maximum quantum yield of U. prolifera. Table S1. qPCR validation of the proteome data. (DOCX $402 \mathrm{~kb}$ )

\section{Acknowledgments}

Not applicable.

\section{Funding}

This work was sponsored by Zhejiang ocean university doctoral research startup funding project (Q1701), University student innovation training program(xj2018011), and the National Natural Science Foundation of China (41606129, 31671009, 41276122). This research was also sponsored by the K.C.Wong Magna Fund in Ningbo University. 


\section{Availability of data and materials}

Please contact author for data requests.

\section{Authors' contributions}

NJX and MHF designed the study. MHF performed proteomics experiments. MHF, XS and ZL participated in the design of the study and performed the statistical analysis. MHF wrote the manuscript with the help of NJX and ZL and JXW. All authors read and approved the final manuscript.

\section{Ethics approval and consent to participate}

Samples of $U$. Prolifera were collected from the intertidal zone of the eastern gulf, Xiangshan, Ningbo, China ( $\left.29^{\circ} 32.803 \mathrm{~N}, 121^{\circ} 45.599 \mathrm{E}\right)$. The Xiangshan municipal government is responsible for this area. The sampling sites were not located within protected areas; however, permission for collection was still obtained. Samples were handled in accordance with guidelines on experimental sample processing for scientific purposes set by of Zhejiang Ocean University, Zhoushan, China.

\section{Consent for publication}

Not applicable.

\section{Competing interests}

All the authors declare that they have no competing interests.

\section{Publisher's Note}

Springer Nature remains neutral with regard to jurisdictional claims in published maps and institutional affiliations.

\section{Received: 4 May 2018 Accepted: 7 October 2018}

\section{Published online: 27 October 2018}

\section{References}

1. Li YX, Zhang XW, Dong X. Differential gene expression in Ulva prolifera under low light and low temperature conditions. Curr Genet. 2012;58(4): 235-44.

2. Wang Y, Wang Y, Zhu L. Comparative studies on the ecophysio-logical differences of two green tide macroalgae under controlled laboratory conditions. PLoS One. 2012;7(8):1-10.

3. Wahid A, Gelani S, Ashraf M, Foolad MR. Heat tolerance in plants: an overview. Environ Exp Bot. 2007:61(3):199-223.

4. Howarth CJ. Genetic improvements of tolerance to high temperature. In: Ashraf M, PJC H, editors. Abiotic stresses: plant resistance through breeding and molecular approaches. New York: Haworth Press Inc.; 2005.

5. Bokszczanin KL, Frargkostefanakis S. Perspectives on deciphering mechanisms underlying plantheat stress response and thermotolerance. Front Plant Sci. 2013;4(315):315-20.

6. Liu GT, Ma L, Duan W, et al. Differential proteomic analysis of grapevine leaves by iTRAQ reveals responses to heat stress and subsequent recovery. BMC Plant Biol. 2014;14(1):110-7.

7. Stirbet A. On the relation between the kautsky effect (chlorophyll a fluorescence induction) and photosystem II: basics and applications of the OJIP fluorescence transient. J Photoch Photobio. 2011;104(1-2):236-57.

8. Fan MH, Sun X, Xu NJ, Liao Z, Wang RX. cDNA cloning, characterization and expression analysis of manganese superoxide dismutase in Ulva prolifera. J Appl Phycol. 2016;28(2):1391-401.

9. Pang QT, Li F, Liu XQ, Wang JT. Compare the growth of Enteromorphaprolifera under different nutrient conditions. Environ Sci. 2013; 34(9):3398-404.

10. Zhu M, Liu ZP, Xu JT, Mao YX, Yao DR. The release, adhesion and germination of spores of Enteromorpha prolifera and its adaptation to different desiccation conditions. Mar Sci. 2011;35(7):1-6.

11. Fan MH, Sun X, Liao Z, Li YH, Wang JX, Fan YP, Cui DL, Li P, Miao ZL. Integration of deep transcriptome and proteome analyses of salicylic acid regulation high temperature stress in Ulva prolifera. Sci Rep. 2017;7(1): 11052-71.

12. Bai YH, Wang S, Zhong $H$, Yang Q, Zhang F, Zhuang ZH, Yuan J, Nie XY, Wang $\mathrm{SH}$. Integrative analyses reveal transcriptome-proteome correlation in biological pathways and secondary metabolism clusters in A. flavusin response to temperature. Scientific Reports. 2015;5:1-13. 5:14582. https://doi. org/10.1038/srep14582.
13. Bai XC, Song H, Lavoie M, Zhu K, Su YY, Ye HQ, Chen S, Fu ZW, Qian HF. Proteomic analyses bring new insights into the effect of a dark stress on lipid biosynthesis in Phaeodactylum tricornutum. Sci Rep. 2016;6:1-10. https://doi.org/10.1038/srep25494.

14. Sun PP, Mao YX, Li GY, Cao M, Kong FN, Wang L, Bi GQ. Comparative transcriptome profiling of Pyropia yezoensis (Ueda) M.S. Hwang \& H.G. Choi in response to temperature stresses. BMC Genomics. 2015;16:463-79.

15. Tia X, Chen L, Wang J, Qiao J, Zhang W. Quantitative proteomics reveals dynamicresponses of Synechocystis sp. PCC 6803 to next-generation biofuel butanol. J Proteome. 2013;78(1):326-45.

16. Qiao J, Wang J, Chen L, Tian X, Huang S, Ren X, Zhang W. Quantitative iTRAQ LC-MS/MS proteomics reveals metabolic responses to biofuel ethanol in cyanobacterial Synechocystis sp.PCC 6803. J Proteome Res. 2012;11(11): $5286-300$.

17. Liu J, Chen L, Wang JX, Qiao J, Zhang W. Proteomic analysis reveals resistance mechanism against biofuel hexane in Synechocystis sp. PCC 6803. Biotechnol Biofuels. 2012;5(1):1-17.

18. Provasoli L. Media and prospect for the cultivation of marine algae, in: Watanabe A, Hattori A (eds) Cultures and collection of algae. Jap Soc Plant Physiol 1968; 63-75.

19. Conesa A, Götz S, García-Gómez JM, Terol J, Talon M, Robles M. Blast2GO: a universal tool for annotation, visualization and analysis in functional genomics research. Bioinformatics. 2005;21(18):3674-6.

20. Du Z, Zhou X, Ling Y, Zhang Z, Su Z. AgriGO: a GO analysis tool kit for the agricultural community. Nucleic Acids Res. 2010;38:W64-70.

21. Emanuelsson $\mathrm{O}$, Nielsen $\mathrm{H}$, Brunak S, Heijne G. Predicting subcellular localization of proteins based on their $\mathrm{N}$-terminal amino acid sequence. J Mol Biol. 2000;300(4):1005-16.

22. Zhang Y, Wang YZ, Li SJ, Zhang XT, Li WH, Luo SX, Sun ZY, Nie RJ. ITRAQbased quantitative proteomic analysis of processed Euphorbia lathyris $L$. for reducing the intestinal toxicity. Proteome Sci. 2018;16:8.

23. Hussain S, Liu GQ, Liu DF, Ahmed M, Hussain N, Teng YW. Study on the expression of dehydrin genes and activities of antioxidative enzymes in oral buds of two sand pear (Pyrus pyrifolia Nakai) cultivars requiring different chilling hours for bud break. Turk J Agric For. 2015;39(6):930-9.

24. Abd El Baky HH, Nofal OA, El Baroty GS. Enhancement of antioxidant enzymes activities, drought stress tolerances and quality of potato plants as response to algal foliar application. Recent Pat Food Nutr Agric. 2016;8(1):70-7.

25. Zhang XY, Hu CG, Yao JL. Tetraploidization of diploid dioscorea results in activation of the antioxidant defense system and increased heat tolerance. J Plant Physiol. 2010;167(2):88-94.

26. Shi JY, Gao LP, Zuo JH, Wang Q, Wang Q, Fan LL. Exogenous sodium nitroprusside treatment of broccoli florets extends shelf life, enhances antioxidant enzyme activity, and inhibits chlorophyll-degradation. Postharvest Biol Tec. 2016;116:98-104.

27. Khare $\mathrm{T}$, Desai $\mathrm{D}$, Kumar $\mathrm{V}$. Effect of $\mathrm{MgCl}_{2}$ stress on germination, plant growth, chlorophyll content, proline content and lipid peroxidation in sorghum cultivars. J Stress Physiol Biochem. 2012;8(4):169-78,

28. Wu HY. Effect of different light qualities on growth, pigment content, chlorophyll fluorescence, and antioxidant enzyme activity in the red alga Pyropia haitanensis (Bangiales Rhodophyta). Biomed Res Int. 2016;7383918:1-8.

29. Brosch M, Yu L, Hubbard T, Choudhary J. Accurate and sensitive peptide identification with mascot percolator. J Proteome Res. 2009:8(6):3176-81.

30. Nguyen THN, Brechenmacher L, Aldrich JT, Clauss TR, Gritsenko MA, Hixson K, Libault M, Tanaka K, Yang F, Yao QM, Lilijana PT, Xu D, Nguyen HT, Stacey G. Quantitative phosphorproteomic analysis ofsoybean root hairs inoculated with radyrhizobium japonicum. Mol Cell Proteomics. 2012;11(11):1140-55.

31. Tolin S, Arrigoni G, Trentin AR, Veljovic-Jovanovic S, Pivato M. Biochemical and quantitative proteomics investigations in Arabidopsis ggt1 mutant leaves reveal a role for the gammaglutamyl cycle in plant's adaptation to environment. Proteomics. 2013;13(12-13):2031-45.

32. Tolin S, Arrigoni G, Moscatiello R, Masi A, Navazio L, Sablok G, Squartini A. Quantitative analysis of the naringenin-inducible proteome in Rhizobium leguminosarum by isobaric tagging and mass spectrometry. Proteomics. 2013;13(12-13):1961-72.

33. Kim HJ, Chiang YH, Kieber JJ, Schaller GE. SCF(KMD) controls cytokinin signaling by regulating the degradation of type-B response regulators. $\mathrm{P}$ Natl Acad Sci USA. 2013:110(24):10028-33.

34. Wang JX, Milton S, Hu Q. Cloning and expression of isoenzymes of superoxide dismutase in Haematococcus pluvialis (Chlorophyceae) under oxidative stress. J Appl Phycol. 2011;23(6):995-1003. 
35. Tiwari V, Patel MK, Chaturvedi AK, Mishra A, Jha B. Functional characterization of the tau class glutathione-s-transferases gene (SbGSTU) promoter of Salicornia brachiata under salinity and osmotic stress. PLoS One. 2016:11(2):e0148494 1-20.

36. Lee KH, Marshall RS, Slivicke LM, Vierstra RD. Genetic analyses of the Arabidopsis 265 proteasome regulatory particle reveal its importance during light stress and a specific role for the N-terminus of RPT2 in development. Plant Signal Behav. 2012;7(8):973-8.

37. Sun XB, Meng XZ, Xu ZK, Song RT. Expression of the 265 proteasome subunit RPN10 is upregulated by salt stress in Dunaliella viridis. J Plant Physiol. 2010;167(12):1003-8.

38. Dittami SM, Scornet D, Petit JL, Ségurens B, Silva CD, Corre E, Dondrup M, Glatting KH, König R, Sterck L, Rouzé P, de Peer YV, Cock JM, Boyen C Tonon T. Global expression analysis of the brown alga Ectocarpus siliculosus (Phaeophyceae) reveals large scale reprogramming of the transcriptome in response to abiotic stress. Genome Biol 2009;10(6):R66.1-R66.20.

39. Tian DQ, Pan XY, Yu YM, Wang WY, Zhang F, Ge YY, Shen XL, Shen FQ, Liu XJ. Denovo characterization of the Anthurium transcriptome and analysis of its digital gene expression under cold stress. BMC Genomics. 2013;14:827-40.

40. Tsai ML, Chang KY, Chiang CS, Shu WY, Weng TC, Chen CR, Huang CL, Lin HK, Hsu IC. UVB radiation induces persistent activation of ribosome and oxidative phosphorylation pathways. Radiat Res. 2009;171:716-24.

41. Li FC, Xu KZ, Ni M, Wang BB, Gu ZY, Shen WD, Li B. Effect of oxidative phosphorylation signaling pathway on silkworm midgut following exposure to phoxim. Environ Toxicol. 2015;32(1):167-77.

42. Reguera M, Peleg Z, Abdel-Tawab YM, Tumimbang EB, Delatorre CA, Blumwald E. Stress-induced cytokinin synthesis increases drought tolerance through the coordinated regulation of carbon and nitrogen assimilation in rice. Plant Physiol. 2013;63(4):1609-22.

43. Rodriguez PL. Protein phosphatase 2 C (PP2C) function in higher plants. Plant Mol Biol. 1998;38(6):919-27.

44. Salvucci ME, Crafts-Brandner SJ. Inhibition of photosynthesis by heat stress: the activation state of rubisco as a limiting factor in photosynthesis. Plant Physiol. 2004;120(2):179-86.

45. Xu Y, Chen CS, Ji DH, Hang N, Xie C. Proteomic profile analysis of Pyropia haitanensis in response to high-temperature stress. J Appl Phycol. 2014; 26(1):607-18.

46. Bassi R, Soen SY, Frank G, Zuber H, Rochaix JD. Characterization of chlorophyll a/b proteins of photosystem I from Chlamydomonas reinhardtii. J Biol Chem. 1992;267:25714-21.

47. Naumann B, Stauber EJ, Busch A, Sommer F, Hippler M. N-terminal processing of Lhca3 is a key step in remodeling of the photosystem I-lightharvesting complex under iron deficiency in Chlamydomonas reinhardtii. J Biol Chem. 2005;280:20431-41.

48. Mozzo M, Mantelli M, Passarini F, Caffarri S, Croce R, Bassi R. Functional analysis of photosystem I light-harvesting complexes (Lhca) gene products of Chlamydomonas reinhardtii. Biochim Biophys Acta. 2010;1797:212-21.

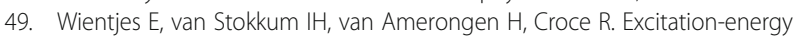
transfer dynamics of higher plant photosystem I light-harvesting complexes. Biophys J. 2011;100(5):1372-80.

50. Zhang T, Gong HM, Wen XG, Lu CM. Salt stress induces a decrease in excitation energy transfer from phycobilisomes to photosystem II but an increase to photosystem I in the cyanobacterium Spirulina platensis. J Plant Physiol. 2010;167(12):951-8.

51. Dong MT, Zhang XW, Zhuang ZM. Characterization of the LhcSR gene under light and temperature stress in the green alga Ulva linza. Plant Mol Biol Rep. 2012;30(1):10-6.

52. Hendrickson L, Sharwood R, Ludwig M, Whitney SM, Badger MR, von Caemmerer $\mathrm{S}$. The effects of rubisco activase on $C_{4}$ photosynthesis and metabolism at high temperature. J Exp Bot. 2008;59(7):1789-98.

53. Nishinaka T, IchijoY IM, Kimura M, Katsuyama M, Iwata K, Miura T, Terada T, Yabe-Nishimura C. Curcumin activates human glutathione S-transferase P1 expression through antioxidant response element. Toxicol Lett. 2007;170(3): 238-47

54. Lyu K, Gu L, Li BP, Lu YC, Wu CC, Guan HY, Yang Z. Stress-responsive expression of a glutathione S-transferase (delta)gene in waterflea daphnia magna challenged by microcystin-producing and microcystin free microcystis aeruginosa. Harmful Algae. 2016;56:1-8.

55. Pflugmacher S, Schwarz S, Pachur HJ, Steinberg CEW. Effects of tributyltin chloride (tbtcll) on detoxication enzymes in aquatic plants. Environ Toxicol2015;15(15):225-233.
56. Deponte M. Glutathione catalysis and the reaction mechanisms of glutathionedependent enzymes. Biochim Biophys Acta. 2013;1830(5):3217-66.

57. Meister A. Glutathione metabolism and its selective modification. J Biol Chem. 1988:263(33):17205-8.

58. Vanacker $\mathrm{H}$, Carver TLW, Foyer $\mathrm{CH}$. Early $\mathrm{H}_{2} \mathrm{O}_{2}$ accumulation in mesophyll cells leads to induction of glutathione during the hyper sensitive response in the barley-powdery mildew interaction. Plant Physiol. 2000;123(4):1289-300.

59. Nawaz Z, Kakar KU, Saand MA, Shu QY. Cyclic nucleotide-gated ion channel gene family in rice, identification, characterization and experimental analysis of expression response to planthormones, biotic and abiotic stresses. BMC Genomics. 2014;15(1):853-60.

60. Jammes F, Hu HC, Villiers F, Bouten R, Kwak JM. Calciumpermeable channels in plant cells. FEBS J. 2011;278(22):4262-76.

61. Qi Z, Verma R, Gehring C, Yamaguchi Y, Zhao YC, Ryan CA, Berkowitzb GA. $\mathrm{Ca}^{2+}$ signaling by plant Arabidopsis thaliana pep peptides depends on AtPepR1, a receptor with guanylyl cyclase activity, and cGMP-activated $\mathrm{Ca}^{2+}$ channels. P Natl Acad Sci USA. 2010;107(49):21193-8.

62. Lodish H, Berk A, Zipursky S. Molecular Cell Biology, vol. 5. 7th ed. New York: Freeman WH and CO; 2013. p. 675.

63. Bazil JN, Beard DA, Vinnakota KC. Catalytic coupling of oxidative phosphorylation, ATP demand, and reactive oxygen species generation. Biophys J. 2016;110:962-7.

64. Bian YW, Deng X, Yan X, Zhou JX, Yuan LL, Yan YM. Integrated proteomic analysis of Brachypodium distachyon roots and leaves reveals a synergistic network in the response to drought stress and recovery. Sci Rep. 2017; 7(46183):1-15
Ready to submit your research? Choose BMC and benefit from:

- fast, convenient online submission

- thorough peer review by experienced researchers in your field

- rapid publication on acceptance

- support for research data, including large and complex data types

- gold Open Access which fosters wider collaboration and increased citations

- maximum visibility for your research: over $100 \mathrm{M}$ website views per year

At BMC, research is always in progress.

Learn more biomedcentral.com/submissions 